\title{
Coalition-Based Cooperative Packet Delivery under Uncertainty: A Dynamic Bayesian Coalitional Game
}

\author{
Khajonpong Akkarajitsakul, Member, IEEE, \\ Ekram Hossain, Senior Member, IEEE, and Dusit Niyato, Member, IEEE
}

\begin{abstract}
Cooperative packet delivery can improve the data delivery performance in wireless networks by exploiting the mobility of the nodes, especially in networks with intermittent connectivity, high delay and error rates such as wireless mobile delay-tolerant networks (DTNs). For such a network, we study the problem of rational coalition formation among mobile nodes to cooperatively deliver packets to other mobile nodes in a coalition. Such coalitions are formed by mobile nodes which can be either well behaved or misbehaving in the sense that the well-behaved nodes always help each other for packet delivery, while the misbehaving nodes act selfishly and may not help the other nodes. A Bayesian coalitional game model is developed to analyze the behavior of mobile nodes in coalition formation in presence of this uncertainty of node behavior (i.e., type). Given the beliefs about the other mobile nodes' types, each mobile node makes a decision to form a coalition, and thus the coalitions in the network vary dynamically. A solution concept called Nash-stability is considered to find a stable coalitional structure in this coalitional game with incomplete information. We present a distributed algorithm and a discrete-time Markov chain (DTMC) model to find the Nash-stable coalitional structures. We also consider another solution concept, namely, the Bayesian core, which guarantees that no mobile node has an incentive to leave the grand coalition. The Bayesian game model is extended to a dynamic game model for which we propose a method for each mobile node to update its beliefs about other mobile nodes' types when the coalitional game is played repeatedly. The performance evaluation results show that, for this dynamic Bayesian coalitional game, a Nash-stable coalitional structure is obtained in each subgame. Also, the actual payoff of each mobile node is close to that when all the information is completely known. In addition, the payoffs of the mobile nodes will be at least as high as those when they act alone (i.e., the mobile nodes do not form coalitions).
\end{abstract}

Index Terms-Wireless mobile networks, delay-tolerant networks, cooperative packet delivery, coalitional game, Bayesian coalitional game, Nash-stable coalitionlal structure, Bayesian core, grand coalition

\section{INTRODUCTION}

$\mathrm{M}$ OBILITY of the nodes can be exploited for data dissemination in wireless mobile networks with intermittent connectivity and very low link reliability such as the wireless mobile delay-tolerant networks (DTNs) [1]. An example scenario is where a base station (e.g., a roadside base station (RBS) or access point) transmits data to the mobile nodes inside its coverage area of transmission and then these mobile nodes carry-and-forward the data to the destination mobile nodes when there is no end-to-end connectivity among the mobile nodes and the base station. Such a cooperative data delivery scheme would be useful for many applications such as vehicular sensor networking, traffic safety, vehicular telematics, and infotainment applications. A few works in the literature proposed cooperative communications models with relay-based mechanisms in DTNs and mobile ad hoc networks to decrease the delay of

- K. Akkarajitsakul and E. Hossain are with the Department of Electrical and Computer Engineering, University of Manitoba, 75A Chancellor's Circle, Winnipeg, MB R3T 5V6, Canada.

E-mail: khajonpong@gmail.com,ekram@ee.umanitoba.ca.

- D. Niyato is with the School of Computer Engineering, Nanyang Technological University, Nanyang Avenue, Singapore 639798.

Manuscript received 25 July 2011; revised 21 Oct. 2011; accepted 4 Nov. 2011; published online 15 Nov. 2011.

For information on obtaining reprints of this article, please send e-mail to: tmc@computer.org, and reference IEEECS Log Number TMC-2011-07-0419. Digital Object Identifier no. 10.1109/TMC.2011.251. data delivery (e.g., [2], [3], [4]). The key assumption in all of the existing schemes is that the mobile nodes which are located near each other always help each other for data delivery. However, due to the tradeoff between performance improvement (i.e., smaller packet delivery delay) and transmission cost (i.e., bandwidth usage and energy consumption), the rational mobile nodes may not always want to help the same other mobile nodes. Consequently, cooperation among the nodes in a group would be dynamic, and the dynamics of the formation of groups among cooperative nodes (or coalitions) needs to be analyzed. In this paper, we use the theory of coalitional games [5], [6], [7] to analyze how the coalitions are formed among mobile nodes for cooperative packet delivery.

As an example, Fig. 1 shows a downlink data communications scenario based on coalition formation where carry-and-forward-based cooperative packet delivery is used to send packets from the base station to mobile nodes. Mobile nodes in the same coalition help each other to deliver packets sent from the base station to the destinations. Here, each rational mobile node makes a decision to leave its current coalition and join another coalition based on its individual preference over coalitions. When the number of mobile nodes in a coalition increases, the packet delivery delay decreases (i.e., due to the fact that there are more mobile nodes to carry and forward the packet) [8].

After the coalitions of mobile nodes are formed, the rational mobile nodes, which are the members of a coalition, 


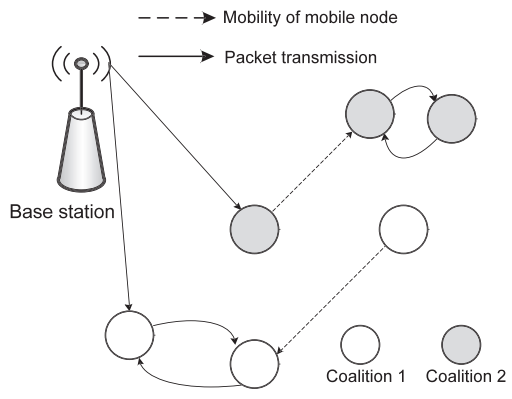

Fig. 1. In a wireless network, mobile nodes can form coalitions to help forward data from a base station to other mobile nodes which are out of the transmission range of the base station.

will agree to always help each other for packet delivery. However, some misbehaving mobile nodes may break the agreement and may not help other nodes (e.g., to reduce their transmission costs [9] and improve their own benefits). As a result, a fully cooperative scenario may not exist. Also, whether a mobile node is well behaved or misbehaving cannot be perfectly known to the other mobile nodes. That is, a mobile node cannot observe whether other mobile nodes will forward packets to third-person or destination mobile nodes or not. Nevertheless, a mobile node can gradually learn other mobile nodes' behaviors. Since each of the mobile nodes will encounter the other mobile nodes in the same coalition, it can estimate the type of a particular node whether it is well behaved or misbehaving by maintaining its own belief based on its experience of interactions with that node.

To model the dynamics of coalition formation under the uncertainty of node behaviors (which is referred to as the types of mobile nodes), we propose a Bayesian coalitional game for coalition-based cooperative packet delivery. In this model, the mobile nodes' observations are used to update their beliefs about other mobile nodes' types and used when the next coalition formation game is played. Therefore, this can be considered as a dynamic Bayesian coalitional game.

The major contributions of the paper can be summarized as follows:

- formulation of a Bayesian coalitional game to model the uncertainty in node behavior for cooperative packet delivery in wireless mobile networks,

- analysis of two solution concepts, namely, Nashstable equilibrium and Bayesian core, for the proposed Bayesian coalitional game,

- extension of the static Bayesian coalitional game to a multistage dynamic coalitional game and proposal of a belief update mechanism for the dynamic Bayesian coalitional game, and

- a comprehensive performance evaluation of the proposed game model.

The rest of this paper is organized as follows: Section 2 describes the system model and assumptions. The Bayesian coalitional game model and the coalition formation algorithm under uncertainty in node behavior are presented in Section 3. Section 4 presents the analysis of the Bayesian coalition formation process. In particular, two solution concepts, namely, the Nash-stable coalitional structure and Bayesian core are analyzed. The dynamic Bayesian coalitional game is presented in Section 5. Section 6 presents the performance evaluation results for the proposed Bayesian coalitional game framework. Section 7 presents the related work and Section 8 concludes the paper.

\section{System Model and Assumptions}

\subsection{Network and Communication Model}

We consider a scenario with $N$ rational mobile nodes which can form coalitions among them for cooperative packet delivery to/from the base stations. We assume that each mobile node will carry-and-forward packets to other mobile nodes in the same coalition when they meet each other. In this scenario, we assume that, over a period of time, the patterns of mobility and interencounter time of each mobile node can be predicted (e.g., by using the technique in [10]). The interencounter interval between nodes is assumed to be exponentially distributed [11]. ${ }^{1}$ Let mobile node $i$ meet another mobile node $j$ with rate $r_{i j}=r_{j i}$ per unit of time. Let $r_{i 0}$ and $r_{0 i}$ denote the rates that mobile node $i$ meets the base station and vice versa. Note that " 0 " is used as the index for a base station. Since the end-to-end connectivity among mobile nodes does not always exist, statistical data about the encounter rates among the mobile nodes are used for estimation of mobility patterns of the nodes [9], [12]. We assume that there is a coordinator at the central application server to collect information about the mobile nodes (e.g., mobility and encounter-rate information).

Any mobile node $i$ receives packets from the base station or from another mobile node $j$ in the same coalition at the cost of $c_{i j}^{\mathrm{r}}$ per packet. ${ }^{2}$ Mobile node $i$ then forwards the packets to their destination or to another mobile node $j^{\prime}$ in the same coalition which does not have these packets. For mobile node $i$, the cost of this transmission is $c_{i j^{\prime}}^{\mathrm{f}}$ per packet. The cost of receiving its own packets by a mobile node, and the cost of packet transmission by a base station are assumed to be zero. Let $d_{i}$ denote the packet delivery delay which is the duration from when a packet is originally transmitted from the base station to when the packet is received by its destination. Given the benefit of smaller delay due to cooperative packet delivery at the cost of relaying packets to the other mobile nodes in the same coalition, a coalitional game-theoretic approach is applied to analyze the coalition formation process among mobile nodes.

\subsection{Uncertainty in Node Behavior for Cooperative Packet Delivery}

We assume that there are two types of mobile nodes, i.e., well behaved node and misbehaving node.

- A well-behaved node always helps to deliver packets to the other nodes in the same coalition.

- A misbehaving node does not always help to deliver packets to other nodes.

In particular, a misbehaving node $i$ may refuse to deliver a packet of other nodes in the same coalition with probability $\varsigma_{i}$. In other words, the probability that a well-behaved node and a misbehaving node will forward the packets of other nodes is 1

1. We verify this assumption later in this paper during performance evaluation.

2. This cost can be defined based on the application as well as the physical transmission parameters. For example, in [13], for packet forwarding in an ad hoc network, the cost was defined in terms of the energy consumption. 
and $1-\varsigma_{i}$, respectively. We assume that a mobile node does not know the types of other mobile nodes. Due to the absence of a monitoring mechanism such as the one in [14], a mobile node cannot observe whether a packet sent to the next mobile node will be forwarded to other mobile nodes or not.

We define a set of cooperative and noncooperative acknowledgements as $A_{\Omega}=\left\{\Omega_{c}, \Omega_{n}\right\}$ and a set of observations of cooperative and noncooperative acknowledgements as $A_{\omega}=\left\{\omega_{c}, \omega_{n}\right\}$. In particular, cooperative acknowledgement (i.e., $\Omega_{c}$ ) and noncooperative acknowledgement (i.e., $\Omega_{n}$ ) mean that a mobile node accepts and refuses, respectively, to help deliver a packet. In other words, when the mobile node sends a packet (i.e., a data packet that will be forwarded) to another mobile node, it implies that a cooperative acknowledgement is done by the mobile node who is a sender. If the mobile node does not send any new packet to another mobile node, it implies that a noncooperative acknowledgement is done by the sending node. Next, we consider two observation errors that can cause imperfect observations in the network.

- False positive observation error, which occurs with probability $p_{e}$, means that a cooperative acknowledgement $\Omega_{c}$ from one mobile node is observed by another mobile node as a noncooperative acknowledgement $\omega_{n}$ due to, for example, link breakage, transmission error, or no new packet to transmit.

- False negative observation error, which occurs with probability $p_{s}$, means that a noncooperative acknowledgement $\Omega_{n}$ corresponding to one mobile node is observed by another mobile node as a cooperative acknowledgement $\omega_{c}$ which is caused by the malicious behavior of a misbehaving node.

When a pair of mobile nodes (e.g., mobile nodes 1 and 2) encounter each other, a connection is initialized and packet transmission starts. Mobile node 2 requests a packet from mobile node 1 and mobile node 2 observes the behavior of mobile node 1 . If mobile node 2 receives a packet from mobile node 1 , mobile node 2 perfectly knows that mobile node 1 helps to forward packets at this time (i.e., mobile node 2 observes $\omega_{c}=\Omega_{c}$ ). If no packet is received by mobile node 2, a noncooperative acknowledgement $\omega_{n}$ is observed. Moreover, if mobile node 1 has a new packet but it does not transmit the packet (i.e., mobile node 1 does a noncooperative acknowledgement $\Omega_{n}$ ), mobile node 2 will definitely not receive any packet and thus observe a noncooperative acknowledgement $\omega_{n}$.

Since mobile node 2 itself directly experiences the packet forwarding from mobile 1 , a false negative observation error (i.e., the event that mobile node 1 does a noncooperative acknowledgement $\Omega_{n}$ but mobile node 2 observes a cooperative acknowledgement $\omega_{c}$ ) cannot occur in our model. In other words, mobile node 2 itself directly experiences only a noncooperative acknowledgement $\omega_{n}{ }^{3}$ However, observation of a noncooperative acknowledgement $\omega_{n}$ may be caused by the event of packet forwarding refusal if mobile node 1 is misbehaving or by the event of false positive observation error if mobile node 1 is well behaved. Then, mobile node 2 cannot conclude whether mobile node 1 is a well-behaved or a misbehaving node. Note that mobile node 2 may not need to actually carry the

3. False negative observation error will not be used later in the analysis. packet after it is received if mobile node 2 is also a misbehaving mobile node.

\subsection{Cooperative Packet Delivery Protocol}

The observations on the other mobile nodes' behaviors are used to estimate the types of other mobile nodes. Then, given the uncertainty of types of other mobile nodes, we obtain the expected payoff function for mobile nodes. The expected payoff is a function of the average cost of communication and the average packet delivery delay. The expected payoffs of all the mobile nodes are used to determine whether the current coalitional structure is stable or not. If it is unstable, a new coalitional structure will be formed, and then the new expected payoff is calculated. Moreover, while mobile nodes are traveling and participating in the cooperative packet delivery with others in the same coalition, they observe other mobile nodes' behavior in cooperative packet delivery. Then, the mobile nodes update their beliefs, i.e., the probabilities of types of other mobile nodes, by using a belief update mechanism. This process is repeated until a stable solution is reached.

The cooperative packet delivery protocol for the mobile nodes to achieve a stable coalition-based solution works as follows:

1. Mobile node $i$ has to be registered to a coordinator at the central application server.

2. Mobile node $i$ submits its information (i.e., rate of encounter $r_{i j}$ with other mobile nodes) to the coordinator.

3. Mobile node $i$ can ask for the information about the other mobile nodes from the coordinator.

4. The mobile nodes play a coalitional game to obtain a stable coalitional structure (i.e., stable groups of mobile nodes participating in cooperative packet delivery).

5. Each mobile node carries and forwards packets to others within the same coalition.

6. Each mobile node observes others' behaviors of packet delivery and updates its beliefs about other mobile nodes' types based on the observations.

7. The mobile nodes repeatedly play the coalitional game.

\section{Formulation of the Bayesian Coalitional GAME MODEL}

We formulate a Bayesian coalitional game model to capture the uncertainty in players' types in coalition formation in an incomplete information environment. This Bayesian coalitional game is similar to a Bayesian noncooperative game [15], [16], [17].

\subsection{Bayesian Coalitional Game with Nontransferable Utility (NTU)}

Definition 1. A Bayesian coalitional game with nontransferable utility is defined as

$$
G=\left\langle\mathbb{N}, \mathbb{T}, \mathcal{P},\left(\bar{u}_{i}\right)_{i \in \mathbb{N}},\left(\succeq_{i}\right)_{i \in \mathbb{N}}\right\rangle .
$$

The formulation of this game is as follows:

- Players. The set of players consists of $N$ rational mobile nodes and is denoted by $\mathbb{N}=\{1, \ldots, N\}$. 
- $\quad$ Type. The type space is denoted by $\mathbb{T}=\mathbb{T}_{1} \times \cdots \times$ $\mathbb{T}_{M}$, where $\mathbb{T}_{i}=\left\{T_{w}, T_{m}\right\}$ denotes a player's possible type set-type $T_{w}$ is for well-behaved nodes and type $T_{m}$ is for misbehaving nodes. Each player $i$ has a type $t_{i} \in \mathbb{T}_{i}$. Each mobile node can observe its own type completely but not the types of other mobile nodes. Each well-behaved mobile node always acts cooperatively with others. That is, it always tries to send or receive packets to or from others when it encounters any other mobile node in the same coalition (i.e., a cooperative acknowledgement $\Omega_{c}$ occurs with probability 1 ). However, a misbehaving mobile node does not always cooperate with others. That is, a misbehaving node refuses to deliver a packet with probability $\varsigma_{i}$ (i.e., a cooperative acknowledgement $\Omega_{c}$ and a noncooperative acknowledgement $\Omega_{n}$ occur with probabilities $1-\varsigma_{i}$ and $\varsigma_{i}$, respectively). Also, the probability $\varsigma_{i}$ for mobile node $i$ is unknown to the other mobile nodes. $t_{0}$ is the type of a base station which is always $T_{w}$ and is perfectly known to all the mobile nodes. Note that a player cannot have both the types at the same time and its type is assumed not to change for a sufficiently long period of time. ${ }^{4}$

- Probability Distribution. $\mathcal{P}$ is a common a priori probability over the type in $\mathbb{T}$. Let $P_{j}^{i}\left(t_{j}=T_{w}\right)=p_{i j}$ and $P_{j}^{i}\left(t_{j}=T_{m}\right)=1-p_{i j}$ denote mobile node $i$ 's belief probabilities about mobile node $j$ over types $T_{w}$ and $T_{m}$, respectively.

- Payoff. $\bar{u}_{i}\left(\mathcal{S}, \overrightarrow{\mathbf{t}}_{\mathcal{S}}^{i}\right)$, is defined as the expected payoff of mobile node $i$ which is the difference between the average utility and the average cost given the beliefs of node $i$ about the types of all players in the coalition $\mathcal{S}$. The vector of types of all players is denoted as $\overrightarrow{\mathbf{t}}_{\mathcal{S}}^{i}=\left[\ldots, t_{j}^{i}, \ldots\right]^{T}, j \in \mathcal{S}$, where $t_{j}^{i}$ is the belief of node $i$ about the type of mobile node $j$, which is a member of coalition $\mathcal{S}$.

With a discrete type space, the expected payoff of node $i$ can be defined as follows [18]:

$$
\begin{aligned}
\bar{u}_{i}\left(\mathcal{S}, \overrightarrow{\mathbf{t}}_{\mathcal{S}}^{i}\right) & =E\left[\alpha_{i} R_{i}\left(\mathcal{S}, \overrightarrow{\mathbf{t}}_{\mathcal{S}}^{i}\right)-\beta_{i} C_{i}\left(\mathcal{S}, \overrightarrow{\mathbf{t}}_{\mathcal{S}}^{i}\right)\right] \\
& =\sum_{k=1}^{2^{N-1}} p_{i}^{\prime k}\left(\overrightarrow{\mathbf{t}}_{\mathcal{S} \backslash\{i\}}^{i}\right)\left(\alpha_{i} R_{i}^{k}\left(\mathcal{S}, \overrightarrow{\mathbf{t}}_{\mathcal{S}}^{i}\right)-\beta_{i} C_{i}^{k}\left(\mathcal{S}, \overrightarrow{\mathbf{t}}_{\mathcal{S}}^{i}\right)\right),
\end{aligned}
$$

where $\alpha_{i}$ and $\beta_{i}$ denote, respectively, the nonnegative weight constants of the average utility and the average cost of delivering a packet to other mobile nodes in the same coalition. Since the type $t_{i}$ of mobile node $i$ is completely known to itself, for node $i$, $p_{i}^{\prime k}\left(\overrightarrow{\mathbf{t}}_{\mathcal{S} \backslash\{i\}}^{i}\right)$ is its joint belief probability about other mobile nodes in the same coalition $\mathcal{S}$ corresponding to the index $k$, and $\overrightarrow{\mathbf{t}}_{\mathcal{S} \backslash\{i\}}^{i}$ is the belief vector of mobile node $i$ about the types of other mobile nodes. For a particular index $k$, the joint belief probability can be expressed as follows:

$$
p_{i}^{\prime}\left(\overrightarrow{\mathbf{t}}_{\mathcal{S} \backslash\{i\}}^{i}\right)=\prod_{j \in \mathcal{S} \backslash\{i\}} P_{j}^{i}\left(t_{j}=t_{j}^{i}\right),
$$

4. If the type of a node changes frequently, during coalition formation, it may not be possible to learn what the type of this node is. where $t_{j}^{i}$ is the type of mobile node $j$ believed by mobile node $i$.

The utility of mobile node $i$ is defined as a function $R_{i}(\mathcal{S})$ as follows:

$$
R_{i}\left(\mathcal{S}, \overrightarrow{\mathbf{t}}_{\mathcal{S}}^{i}\right)= \begin{cases}\max \left(0,1-\frac{d_{i}(\mathcal{S})}{\min \left(\hat{d}_{i}, d_{i}^{T T L}\right)}\right), & |\mathcal{S}|>1 \\ 0, & \text { otherwise }\end{cases}
$$

where $d_{i}(\mathcal{S})$ is the packet delivery delay for mobile node $i \in \mathcal{S}, \hat{d}_{i}=d_{i}(\{i\})$ is the packet delivery delay when mobile node $i$ acts alone, and $d_{i}^{T T L}$ is the timeto-live (TTL) value for a packet.

The average cost of mobile node $i$ for delivering a packet to any mobile node $j$ in the same coalition can be expressed as follows:

$$
C_{i}\left(\mathcal{S}, \overrightarrow{\mathbf{t}}_{\mathcal{S}}^{i}\right)= \begin{cases}\sum_{j \in \mathcal{S}, j \neq i} c_{i j}(\mathcal{S}), & |\mathcal{S}|>1 \\ 0, & \text { otherwise }\end{cases}
$$

where $c_{i j}(\mathcal{S})$ is the average cost that mobile node $i$ incurs for delivering a packet destined to mobile node $j$ in the same coalition $\mathcal{S}$ and $|\mathcal{S}|$ is the number of mobile nodes in coalition $\mathcal{S}$. In Appendix A, which can be found on the Computer Society Digital Library at http://doi.ieeecomputersociety.org/ 10.1109/TMC.2011.251, ${ }^{5}$ we formulate a Markov chain model to find the utility and the average cost (and hence the expected payoff) of each mobile node under uncertainty about other mobile nodes' types.

- Preference. $\succeq_{i}$ describes player $i$ 's preference. For example, $\mathcal{S}_{1} \succeq_{i} \mathcal{S}_{2}$ means that player $i$ prefers to be a member of coalition $\mathcal{S}_{2}$ at most as much as $\mathcal{S}_{1}$.

- Action. The action of each player is to make a decision on which coalition to form (i.e., to join or leave a coalition) based on its own payoff and the payoffs of other players in the current coalition as well as the new coalition.

Each of the well-behaved mobile nodes always helps others by sending cooperative acknowledgements and doing cooperation. However, due to a false positive observation error which occurs with probability $0<p_{e}<1$, a mobile node imperfectly observes other mobile nodes' behaviors. Note that a false negative observation error occurs with probability $p_{s}=0$.

In this paper, we consider a nontransferable utility coalitional game since the individual payoff of each mobile node (i.e., utility as a function of packet delivery delay minus cost of helping other nodes to deliver packets) cannot be given or transferred arbitrarily to other mobile nodes. The solution of the coalitional game is a stable coalitional structure.

\subsection{Coalition Formation}

Definition 2. A coalitional structure is a set of coalitions spanning all the users in $\mathbb{N}$ which is defined as $\Upsilon=\left\{\mathcal{S}_{1}, \ldots, \mathcal{S}_{l}, \ldots, \mathcal{S}_{S}\right\}$, where $\mathcal{S}_{l} \cap \mathcal{S}_{l}^{\prime}=\emptyset$ for $l \neq l^{\prime}$ and $S$ is the total number of

5. See the online supplementary file. 
coalitions for $1 \leq S \leq N$, and $\bigcup_{l=1}^{s} \mathcal{S}_{l}=\mathbb{N}$. The coalition consisting of all the mobile nodes is referred to as a grand coalition. There can be $2^{N}-1$ distinct nonempty coalitions and $D_{N}$ different coalitional structures for $N$ players, where $D_{N}$ is the Nth Bell number given as follows:

$$
D_{N}=\sum_{j=0}^{N-1}\left(\begin{array}{c}
N-1 \\
j
\end{array}\right) D_{j}, \quad \text { for } \quad N \geq 1 \quad \text { and } \quad D_{0}=1 .
$$

Let $\mathcal{S}_{l}^{i}$ denote any coalition $\mathcal{S}_{l}$ for $i \in \mathcal{S}_{l}$. In a coalitional game, a player's action is to choose a coalition that the player prefers to be a member of according to its expected payoff. Therefore, the concept of preference has to be defined.

Definition 3. The preference of any mobile node $i$ is denoted by $\left(\succeq_{i}\right)_{i \in \mathcal{S} \subset \mathbb{N}} \cdot \mathcal{S}_{l}^{i} \succeq_{i} \mathcal{S}_{l^{\prime}}^{i}$ denotes that mobile node $i$ weakly prefers to be a member of $\mathcal{S}_{l}^{i}, i \in \mathcal{S}_{l} \subseteq \mathbb{N}$ over $\mathcal{S}_{l^{\prime}}^{i}, i \in \mathcal{S}_{l^{\prime}} \subseteq \mathbb{N}$ or at least, mobile node $i$ prefers to be a member of both the coalitions. $\mathcal{S}_{l}^{i} \succ_{i} \mathcal{S}_{l^{\prime}}^{i}$ denotes that mobile node $i$ strictly prefers to be a member of $\mathcal{S}_{l}^{i}$ over $\mathcal{S}_{l^{\prime}}^{i}$.

Since a player is rational and the expected payoff of a player in a coalition depends only on the members of the coalition and can be predicted, this game can be considered a hedonic game which is a special case of NTU game [19]. From the definition of hedonic game, the preference of a node can be defined as follows:

- $\mathcal{S}_{l}^{i} \succ_{i} \mathcal{S}_{l^{\prime}}^{i}$ is valid if two following conditions are true. First, all the other mobile nodes $j$ in $\mathcal{S}_{l}^{i}$ believe that they are not worse off when mobile node $i$ is a member of $\mathcal{S}_{l}^{i}$ (i.e., $\left.\bar{u}_{j}\left(\mathcal{S}_{l}^{i}, \overrightarrow{\mathbf{t}}_{\mathcal{S}_{l}^{i}}^{j}\right) \geq \bar{u}_{j}\left(\mathcal{S}_{l}^{i} \backslash\{i\}, \overrightarrow{\mathbf{t}}_{\mathcal{S}_{l}^{j} \backslash\{i\}}\right), \forall j \in \mathcal{S}_{l}^{i} \backslash\{i\}\right)$. Second, mobile node $i$ believes that its payoff, when this node is a member of $\mathcal{S}_{l}^{i}$, is greater than that when this node is a member of $\mathcal{S}_{l^{\prime}}^{i}$ (i.e., $\bar{u}_{i}\left(\mathcal{S}_{l}^{i}, \overrightarrow{\mathbf{t}}_{\mathcal{S}_{l}^{i}}^{i}\right)>$ $\bar{u}_{i}\left(\mathcal{S}_{l^{\prime}}^{i}, \overrightarrow{\mathbf{t}}_{\mathcal{S}_{l^{\prime}}^{i}}^{i}\right)$.

- $\quad \mathcal{S}_{l}^{i} \succeq_{i} \mathcal{S}_{l^{\prime}}^{i^{\prime}}$ is valid if two following conditions are true. First, all the other mobile nodes $j$ in $\mathcal{S}_{l}^{i}$ believe that they are not worse off when mobile node $i$ is a member of $\mathcal{S}_{l}^{i}$ (i.e., $\bar{u}_{j}\left(\mathcal{S}_{l}^{i}, \overrightarrow{\mathbf{t}}_{\mathcal{S}_{l}^{i}}^{j}\right) \geq \bar{u}_{j}\left(\mathcal{S}_{l}^{i} \backslash\{i\}, \overrightarrow{\mathbf{t}}_{\mathcal{S}_{l}^{j} \backslash\{i\}}\right)$, $\left.\forall j \in \mathcal{S}_{l}^{i} \backslash\{i\}\right)$. Second, mobile node $i$ believes that its payoff, when this node is a member of $\mathcal{S}_{l}^{i}$, is not less than that when this node is a member of $\mathcal{S}_{l^{\prime}}^{i}$ (i.e., $\left.\bar{u}_{i}\left(\mathcal{S}_{l}^{i}, \overrightarrow{\mathbf{t}}_{\mathcal{S}_{l}^{i}}^{i}\right) \geq \bar{u}_{i}\left(\mathcal{S}_{l^{\prime}}^{i}, \overrightarrow{\mathbf{t}}_{\mathcal{S}_{l^{\prime}}^{i}}^{i}\right)\right)$, or

- $\quad \mathcal{S}_{l}^{i} \succeq_{i} \mathcal{S}_{l^{\prime}}^{i}$ is valid if the following condition is true. At least one of the other mobile nodes $j$ in $\mathcal{S}_{l^{\prime}}^{i}$ believes that it is worse off when mobile node $i$ is a member of $\mathcal{S}_{l^{\prime}}^{i}$ and no mobile node $j$ in $\mathcal{S}_{l}^{i}$ believes that it is worse off when mobile node $i$ is a member of $\mathcal{S}_{l}^{i}$, or at least one of the other mobile nodes $j$ in both $\mathcal{S}_{l}^{i}$ and $\mathcal{S}_{l^{\prime}}^{i}$ believes that it is worse off when mobile node $i$ is a member of $\mathcal{S}_{l}^{i}$ (i.e., $\bar{u}_{j}\left(\mathcal{S}_{l^{\prime}}^{i}, \overrightarrow{\mathbf{t}}_{\mathcal{S}_{l^{\prime}}^{j}}^{j}\right)<\bar{u}_{j}\left(\mathcal{S}_{l^{\prime}}^{i} \backslash\{i\}, \overrightarrow{\mathbf{t}}_{\mathcal{S}_{l^{\prime}}^{j} \backslash\{i\}}\right), \exists j \in \mathcal{S}_{l^{\prime}}^{i} \backslash\{i\}$ and $\bar{u}_{j}\left(\mathcal{S}_{l}^{i}, \overrightarrow{\mathbf{t}}_{\mathcal{S}_{l}^{j}}^{j}\right)<\bar{u}_{j}\left(\mathcal{S}_{l}^{i} \backslash\{i\}, \overrightarrow{\mathbf{t}}_{\mathcal{S}_{l}^{j} \backslash\{i\}}^{j}\right), \nexists j$ or $\left.\exists j \in \mathcal{S}_{l}^{i} \backslash\{i\}\right)$.

\subsection{Coalition Formation Algorithm}

At any time $\phi$, any single mobile node in a coalition can decide to leave its current coalition and/or join a new

coalition (i.e., make an individual decision). We present a distributed algorithm (Algorithm 1) based on mobile nodes' strict preferences as presented in Definition 3 to achieve a solution of the game.

Algorithm 1. Distributed coalition formation algorithm based on individual preferences for cooperative packet delivery

1: Initialize $\phi=0$ and $\Upsilon(\phi)=\left\{\mathcal{S}_{1}(\phi), \ldots, \mathcal{S}_{s}(\phi)\right\}$

2: loop

3: At time $\phi$, mobile node $i$ is randomly selected to make a decision to leave $\mathcal{S}_{l}^{i}(\phi)$ and join any coalition $\mathcal{S}_{k} \in \Upsilon(\phi) \backslash \mathcal{S}_{l}^{i}(\phi) \cup\{\emptyset\}$.

4: $\quad$ Mobile node $i$ computes its expected payoff $\bar{u}_{i}\left(S_{l}^{i}(\phi), \overrightarrow{\mathbf{t}}_{S_{l}^{i}(\phi)}^{i}\right)$

5: Mobile node $i$ randomly selects one of the coalitions, i.e., $\mathcal{S}_{k}$, to join.

6: $\quad$ Mobile node $i$ computes its expected payoff $\bar{u}_{i}\left(\mathcal{S}_{k}(\phi) \cup\{i\}, \overrightarrow{\mathbf{t}}_{\mathcal{S}_{k}(\phi) \cup\{i\}}^{i}\right)$

7: $\quad$ if $\bar{u}_{i}\left(\mathcal{S}_{k}(\phi) \cup\{i\}, \overrightarrow{\mathbf{t}}_{\mathcal{S}_{k}(\phi) \cup\{i\}}^{i}\right)>\bar{u}_{i}\left(S_{l}^{i}(\phi), \overrightarrow{\mathbf{t}}_{S_{l}^{i}(\phi)}^{i}\right)$

8: $\quad$ Mobile node $i$ sends its request to the central coordinator to join $\mathcal{S}_{k}(\tau)$.

9: $\quad$ Mobile node $j \in \mathcal{S}_{k}(\phi)$ computes and sends its expected payoff $\bar{u}_{j}\left(\mathcal{S}_{k}(\phi) \cup\{i\}, \overrightarrow{\mathbf{t}}_{\mathcal{S}_{k}(\phi) \cup\{j\}}^{j}\right)$ to the central coordinator.

Note that if there is no change of information (e.g., beliefs about the types of other mobile nodes, and delivery costs) that causes any payoff to change, no new calculation or update is required.

10:

11:

12:

13:

$14:$

15:

16: else

17: $\Upsilon(\phi+1)=\Upsilon(\phi)$

18: end

19: $\phi=\phi+1$

20: end loop when a Nash-stable coalitional structure $\Upsilon^{*}$ is obtained (i.e., no mobile node prefers to move to another coalition).

Algorithm 1 works as follows: first, the time $\phi$ is initialized to be zero, and also the coalitional structure is initialized. The algorithm repeats the following steps. Any mobile node $i$ makes a decision to leave or to join the new coalition. To do so, the mobile node has to compute its expected payoff. Given the calculated expected payoff, the mobile node randomly selects a coalition to join. After joining, the mobile node recalculates its expected payoff. If the new expected payoff is higher, the mobile node requests to join the new coalition by sending request message to the coordinator. Upon receiving the request message, the mobile nodes in the target coalition evaluate their expected payoffs in the case that mobile node $i$ joins their coalition. If the expected payoff is higher, the new coalition will be formed. Otherwise, there is no change of the coalitional structure. 
Next, we consider whether any coalitional structure obtained from Algorithm 1 is Nash-stable. The definition of a Nash-stable coalitional structure is given as follows:

Definition 4. A coalitional structure $\Upsilon=\left\{\mathcal{S}_{1}, \ldots, \mathcal{S}_{l}, \ldots, \mathcal{S}_{s}\right\}$ is Nash-stable if $\forall i \in \mathbb{N}, \mathcal{S}_{l}^{i} \succeq_{i} \mathcal{S}_{k} \cup\{i\}$ for all $\mathcal{S}_{k} \in \Upsilon \backslash \mathcal{S}_{l}^{i} \cup$ $\{\emptyset\}$ [19].

From the definition, if a coalitional structure (i.e., a set of coalitions) is stable, then

- No player $i$ has an incentive to leave its current coalition $\mathcal{S}_{l}^{i}$ and act alone (i.e., $\mathcal{S}_{l}^{i} \succeq_{i}\{i\}$ ). This implies that no player believes that she will be better off (in terms of expected payoff) by acting alone, i.e., $\bar{u}_{i}\left(\mathcal{S}_{l}, \overrightarrow{\mathbf{t}}_{\mathcal{S}_{l}}^{i}\right) \geq u_{i}\left(\{i\}, t_{i}\right)$.

- Given a player's beliefs about the other players, no player $i$ will have an incentive to move from its current coalition $\mathcal{S}_{l}^{i}$ to any other coalition (assuming that the other coalitions do not change) that makes the coalitional structure to change (i.e., $\mathcal{S}_{l}^{i} \succeq_{i} \mathcal{S}_{l^{\prime}} \cup$ $\{i\}, \mathcal{S}_{l^{\prime}} \in \Upsilon \backslash \mathcal{S}_{l^{\prime}}^{i}, i \in \mathbb{N}$ and $\left.\mathcal{S}_{l^{\prime}} \succ_{j} \mathcal{S}_{l^{\prime}} \cup\{i\}, \forall j \in \mathcal{S}_{l^{\prime}}\right)$. This implies no player believes that she will be better off by joining the new coalition $\mathcal{S}_{l^{\prime}}$ without making all the players in the coalition $\mathcal{S}_{l^{\prime}}$ believe that they will be worse off, i.e.,

$$
\bar{u}_{i}\left(\mathcal{S}_{l}^{i}, \overrightarrow{\mathbf{t}}_{\mathcal{S}_{l}^{i}}^{i}\right) \geq \bar{u}_{i}\left(\mathcal{S}_{l^{\prime}} \cup\{i\}, \overrightarrow{\mathbf{t}}_{\mathcal{S}_{l^{\prime}} \cup\{i\}}^{i}\right), i \in \mathbb{N}
$$

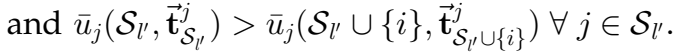

In the next section, we analyze the stability of the Bayesian coalitional game.

\section{Analysis of the Bayesian Coalitional Game}

The concepts of Nash-stability and core [20], [21] are used to analyze the stability of the Bayesian coalition formation game. Also, a discrete-time Markov chain (DTMC) model is developed to analyze the coalition formation algorithm. Note that since the preferences of players are based on their expected payoffs given their beliefs about other players' types, the Nash-stability may be described as the Bayesian Nash-stability which is comparable to the Bayesian Nash equilibrium of a noncooperative game with incomplete information.

\subsection{Bayesian Nash-Stability}

Theorem 1. Algorithm 1 will converge to a Nash-stable coalitional structure $\Upsilon^{*}$.

Proof. From Definition 3, any mobile node will be able to move to another coalition only when none of the mobile nodes in that coalition is worse off (i.e., the mobile nodes believe that they will not be worse off in terms of expected payoff after the new node joins). Starting with any coalitional structure $\Upsilon$, if any mobile node $i$ still prefers to move to a new coalition based on Definition 3 (i.e., $\mathcal{S}_{k} \cup\{i\} \succ_{i} \mathcal{S}_{l}^{i}, \mathcal{S}_{k} \in \Upsilon \backslash \mathcal{S}_{l}^{i} \cup\{\emptyset\}$ ), then the current coalitional structure is not Nash-stable. Consequently, the current coalitional structure changes to a new coalitional structure after mobile node $i$ joins a new coalition (i.e., lines 10-12 of Algorithm 1). Since there can be $2^{N}-1$ distinct nonempty coalitions and $D_{N}$ different coalitional structures as given in (6), this implies that there are maximum $2^{N-1}$ coalitions including an empty coalition for each mobile node $i$ to possibly join. The worst case is that if mobile node $i$ cannot find any nonempty coalition (i.e., the number of members of the coalition is greater than zero, $\left.\left|\mathcal{S}_{k}\right|>0\right)$ to join, mobile node $i$ then forms its singleton coalition.

Note that mobile node $i$ moving to the empty coalition from its current singleton coalition (i.e., currently it is not a member of any other coalition) is considered to have no incentive to deviate from the current coalition because there would be no change in the coalition structure (i.e., it is still the same singleton coalition). Since the number of coalitions that each mobile node $i$ can be a member of is finite, the algorithm will converge to a Nash-stable coalitional structure $\Upsilon^{*}$.

From [19], a Nash-stable coalitional structure is also an individually stable coalitional structure (i.e., Nash-stability is a subset of individual stability).

Definition 5. A coalitional structure $\Upsilon=\left\{\mathcal{S}_{1}, \ldots, \mathcal{S}_{l}, \ldots, \mathcal{S}_{s}\right\}$ is individually stable if $\nexists i \in \mathbb{N}, \nexists \mathcal{S}_{k} \in \Upsilon \backslash \mathcal{S}_{l}^{i} \cup\{\emptyset\}$ such that $\mathcal{S}_{k} \cup\{i\} \succ_{i} \mathcal{S}_{l}^{i}$ and $\forall j \in \mathcal{S}_{k}, \mathcal{S}_{k} \cup\{i\} \succeq_{j} \mathcal{S}_{k}$.

This definition means that no player can move to another coalition, which it prefers to join, without making some members of that coalition worse off. Moreover, we can observe a more specific example of the condition of existence of an individually stable and also Nash-stable coalitional structure which does not consist of any singleton coalition. In particular, no player will leave its current coalition and join an empty coalition (i.e., no singleton coalition will be formed) if the following proposition is true.

Proposition 1. If for all coalitions $\mathcal{S} \subseteq \mathbb{N}$, the condition $\forall i \in$ $\mathcal{S}, \mathcal{S} \succ_{i}\{i\}$ is true, then there exists at least one Nash-stable and also individual stable coalition structure in which all coalitions in the coalition structure are not singleton coalitions.

Proof. This observation states that no player forms a singleton coalition since each player believes that a better expected payoff can be obtained by being a member of a coalition. Since the payoff of a player will be zero if the player acts alone, from (2), we can show that $u_{i}\left(\{i\}, t_{i}\right)=$ $0<\bar{u}_{i}\left(\mathcal{S}, \overrightarrow{\mathbf{t}}_{\mathcal{S}}^{i}\right)$ (i.e., $\left.\mathcal{S} \succ_{i}\{i\}\right), \forall i \in \mathcal{S}$. According to Algorithm 1 , we can obtain a Nash-stable and individual stable coalitional structure at the end.

Since there can be multiple Nash-stable coalition structures, we formulate a discrete-time Markov chain to analyze the Nash-stable coalitional structure [22] resulting from the distributed algorithm.

\subsection{Discrete-Time Markov Chain-Based Analysis of Coalition Formation}

The DTMC follows Algorithm 1 when the state (i.e., coalitional structure) changes based on individual preferences of the players. As an example, with three players, the state transition diagram of the DTMC for coalition formation is shown in Fig. 2.

The state space of the DTMC can be expressed as follows:

$$
\Theta=\left\{\left(\Upsilon_{1}\right), \ldots,\left(\Upsilon_{x}\right), \ldots,\left(\Upsilon_{D_{N}}\right)\right\},
$$

where $\Upsilon_{x}$ is a coalitional structure and $D_{N}$ is the $N$ th Bell number. The transition probability of this DTMC is denoted 


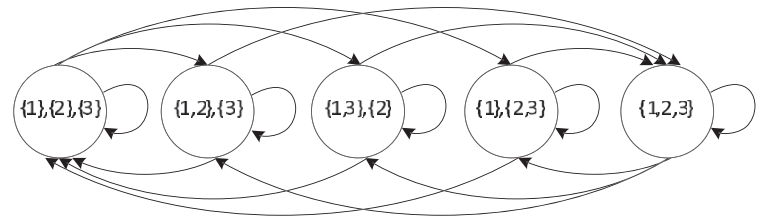

Fig. 2. State transition diagram of the discrete-time Markov chain for coalition formation among three players.

by $\xi_{\Upsilon, \Upsilon^{\prime}}$. In particular, $\xi_{\Upsilon, \Upsilon^{\prime}}$ is the probability that the coalitional structure changes from $\Upsilon$ to $\Upsilon^{\prime}$. Let $\mathcal{B}_{\Upsilon, \Upsilon^{\prime}}$ denote the set of players involved in the change of coalitional structure from $\Upsilon$ to the coalitional structure $\Upsilon^{\prime}$. For example, players 1 and 2 are involved in the change of the coalitional structure from $\Upsilon=\{\{1\},\{2\},\{3\}\}$ to $\Upsilon^{\prime}=$ $\{\{1,2\},\{3\}\}$. The transition probability from state $\Upsilon$ to $\Upsilon^{\prime}$ is then found as follows:

$$
\begin{aligned}
& \xi_{\Upsilon, \Upsilon^{\prime}}= \\
& \left\{\begin{array}{l}
\sum_{i \in \mathcal{B}_{\Upsilon, \Upsilon^{\prime}}} \frac{1}{N} \frac{1}{\left|\Upsilon \backslash \mathcal{S}_{l}^{i} \cup\{\emptyset\}\right|} \varphi_{i}\left(\Upsilon^{\prime} \mid \Upsilon\right), \\
\Upsilon \neq \Upsilon^{\prime} \& \Upsilon^{\prime}=\left(\Upsilon \backslash\left\{\mathcal{S}_{l}^{i}, \mathcal{S}_{k}\right\}\right) \cup\left\{\mathcal{S}_{k} \cup\{i\}\right\} \cup\left\{\mathcal{S}_{l}^{i} \backslash\{i\}\right\} \\
0, \quad \Upsilon \neq \Upsilon^{\prime} \& \Upsilon^{\prime} \neq\left(\Upsilon \backslash\left\{\mathcal{S}_{l}^{i}, \mathcal{S}_{k}\right\}\right) \cup\left\{\mathcal{S}_{k} \cup\{i\}\right\} \cup\left\{\mathcal{S}_{l}^{i} \backslash\{i\}\right\}
\end{array}\right. \\
& \begin{array}{l}
1-\sum_{\Upsilon^{\prime \prime} \in \Theta, \Upsilon^{\prime \prime} \neq \Upsilon} \xi_{\Upsilon, \Upsilon^{\prime \prime}}, \quad \Upsilon=\Upsilon^{\prime},
\end{array}
\end{aligned}
$$

where $\mathcal{S}_{k} \in \Upsilon \backslash \mathcal{S}_{l}^{i} \cup\{\emptyset\}, \frac{1}{N}$ is the probability that player $i$ is selected to make her individual decision, $\frac{1}{|\Upsilon| \mathcal{S}_{l}^{i} \cup\{\emptyset\} \mid}$ is the probability that player $i$ selects one possible coalition $\mathcal{S}_{k} \in$ $\Upsilon \backslash \mathcal{S}_{l}^{i} \cup\{\emptyset\}$ to join. $\varphi_{i}\left(\Upsilon^{\prime} \mid \Upsilon\right)$ is the probability that a player decides to move from its current coalition $\mathcal{S}_{l}^{i}$ to a new coalition $\mathcal{S}_{l^{\prime}}^{i}$ which makes the coalitional structure to change from $\Upsilon$ to $\Upsilon^{\prime}$, i.e.,

$$
\varphi_{i}\left(\Upsilon^{\prime} \mid \Upsilon\right)= \begin{cases}1, & \mathcal{S}_{l^{\prime}}^{i} \succ_{i} \mathcal{S}_{l}^{i} \\ 0, & \text { otherwise, }\end{cases}
$$

where $\mathcal{S}_{l}^{i} \in \Upsilon$ and $\mathcal{S}_{l^{\prime}}^{i} \in \Upsilon^{\prime}$.

Given the transition matrix $\mathbf{Q}$, whose elements are $\xi_{\Upsilon, \Upsilon^{\prime}}$, the stationary probability vector $\vec{\pi}$ can be obtained by solving the following equation: $\vec{\pi}^{T} \mathbf{Q}=\vec{\pi}^{T}$, where $\vec{\pi}^{T} \overrightarrow{\mathbf{1}}=1$, and $\overrightarrow{\mathbf{1}}$ is a vector of ones. $\overrightarrow{\boldsymbol{\pi}}=\left[\begin{array}{lllll}\pi_{\Upsilon_{1}} & \ldots & \pi_{\Upsilon_{x}} & \ldots & \pi_{\Upsilon_{D_{N}}}\end{array}\right]^{T}$, where $\pi_{\Upsilon_{x}}$ is the probability that the coalitional structure $\Upsilon_{x}$ will be formed.

\subsection{Bayesian Core}

In this solution concept, the core is regarded as a set of payoffs corresponding to a grand coalition upon which no other coalition can improve, and therefore, no player has an incentive to leave the grand coalition. The grand coalition refers to a coalition of all the $N$ players, i.e., all mobile nodes participating in the game. We study the conditions to achieve the core of the Bayesian coalitional game (i.e., Bayesian core).

Let $\overrightarrow{\mathbf{u}}^{\mathbb{N}}=\left[\bar{u}_{1}\left(\mathbb{N}, \overrightarrow{\mathbf{t}}_{\mathbb{N}}^{1}\right), \ldots, \bar{u}_{i}\left(\mathbb{N}, \overrightarrow{\mathbf{t}}_{\mathbb{N}}^{i}\right), \ldots, \bar{u}_{N}\left(\mathbb{N}, \overrightarrow{\mathbf{t}}_{\mathbb{N}}^{N}\right)\right]$ and $\overrightarrow{\mathbf{u}}^{\mathcal{S}}=\left[\ldots, \bar{u}_{i}\left(\mathcal{S}, \overrightarrow{\mathbf{t}}_{\mathcal{S}}^{i}\right), \ldots\right]$ denote the expected payoff vectors of all the mobile nodes when they are members in the grand coalition and the expected payoff vectors of the mobile nodes in any coalition $\mathcal{S} \subseteq \mathbb{N}$ calculated from (2), respectively.
Definition 6. The characteristic function of coalition $\mathcal{S}$, which is a set of feasible payoff vector $\overrightarrow{\mathrm{x}}^{\mathcal{S}}$ of length $|\mathcal{S}|$, is defined as follows:

$$
V(\mathcal{S})=\left\{\overrightarrow{\mathbf{x}}^{\mathcal{S}} \in \mathbb{R}^{\mathcal{S}} \mid \overrightarrow{\mathbf{x}}^{\mathcal{S}} \leq \overrightarrow{\overrightarrow{\mathbf{u}}}^{\mathcal{S}}\right\} .
$$

We can use the concepts of core and Bayesian core in the nontransferable utility game [20], [21] and the transferable utility game [15], [16], [17], respectively, to define the Bayesian core in NTU game in Definitions 7 and 8.

Definition 7. For an NTU Bayesian coalitional game, the weak Bayesian core is defined as follows:

$\mathcal{C}=\left\{\overrightarrow{\mathbf{u}}^{\mathbb{N}} \in V(\mathbb{N}) \mid \forall \mathcal{S} \subseteq \mathbb{N}, \nexists \overrightarrow{\mathbf{u}}^{\mathcal{S}} \in V(\mathcal{S})\right.$ s.t. $\left.\mathcal{S} \succeq_{i} \mathbb{N}, \forall i \in \mathcal{S}\right\}$.

This definition indicates that there exist payoffs from the grand coalition upon which no other coalition can improve so that no member in the grand coalition believes that she is better off by deviating from the grand coalition (i.e., no other coalition blocks the grand coalition and its payoffs).

Remark 1. Clearly, in our game model, the coalitional structure composed of only the grand coalition can be a Nash-stable coalitional structure but its expected payoffs may not be the Bayesian core (i.e., the grand coalition can be blocked by other coalitions). On the other hand, if the Bayesian core is not empty, then the coalitional structure composed of only the grand coalition exists and is Nash-stable.

Next, we consider another stability concept called strong Bayesian core. Let $\bar{u}_{j}^{i}\left(\mathcal{S}, \overrightarrow{\mathbf{t}}_{\mathcal{S}}^{i}\right)$ and $\succeq_{j}^{i}$ be the expected payoff vector of mobile node $j$ and the preference of mobile node $j$ believed by mobile node $i$, respectively, when they are members in any coalition $\mathcal{S}$. Note that, $\bar{u}_{i}^{i}=\bar{u}_{i}$, as shown in (2), and $\bar{u}_{j}^{i}$, for $j \neq i$, can be calculated based on the types of other mobile nodes believed by mobile node $i$ as follows:

$$
\bar{u}_{j}^{i}\left(\mathcal{S}, \overrightarrow{\mathbf{t}}_{\mathcal{S}}^{i}\right)=E\left[\alpha_{j} R_{j}\left(\mathcal{S}, \overrightarrow{\mathbf{t}}_{\mathcal{S}}^{i}\right)-\beta_{j} C_{j}\left(\mathcal{S}, \overrightarrow{\mathbf{t}}_{\mathcal{S}}^{i}\right)\right] .
$$

Then, the preference of mobile node $j$ believed by mobile node $i$ can be found by following Definition 3 and using (12) to find the expected payoffs.

Definition 8. For an NTU Bayesian coalitional game, the strong Bayesian core is defined as follows:

$$
\begin{aligned}
\mathcal{C}= & \left\{\overrightarrow{\mathbf{u}}^{\mathbb{N}} \in V(\mathbb{N}) \mid \forall \mathcal{S} \subseteq \mathbb{N}, \nexists \overrightarrow{\mathbf{u}}^{\mathcal{S}} \in V(\mathcal{S})\right. \\
& \text { s.t. } \left.\mathcal{S} \succeq_{i} \mathbb{N}, \forall i \in \mathcal{S} \text { and } \mathcal{S} \succeq_{j}^{i} \mathbb{N}, \forall i \in \mathcal{S}, \forall j \in \mathcal{S}, j \neq i\right\} .
\end{aligned}
$$

The definition states that there exist payoffs from the grand coalition such that, no member in the coalition believes that she is better off by leaving the grand coalition, and each member, who uses her own view of expected payoffs of others members, believes that no other member is better off if she leaves the grand coalition. The strong Bayesian core is a subset of the weak Bayesian core. Therefore, if the weak Bayesian core exists, it may or may not be the strong Bayesian core. In our NTU game, the strong Bayesian core cannot be considered if all the needed information about the other mobile nodes (i.e., weight constants and costs of packet delivery) except their actual 
TABLE 1

Solution Concepts and the Corresponding Conditions for the Proposed Cooperative Packet Delivery Game

\begin{tabular}{|l|l|}
\hline Solution concept & Condition \\
\hline Nash stability & $\begin{array}{l}\text { 1) No player has an incentive to leave its current coalition and act alone. } \\
\text { 2) No player will have an incentive to move from its current coalition to any other coalition (assuming that } \\
\text { the other coalitions do not change) that makes the coalitional structure to change. }\end{array}$ \\
\hline Individual stability & $\begin{array}{l}\text { No player can move to another coalition, which it prefers to join, without making some members of that } \\
\text { coalition worse off. }\end{array}$ \\
\hline Weak Bayesian core & $\begin{array}{l}\text { There exist payoffs from the grand coalition upon which no other coalition can improve so that no member } \\
\text { in the grand coalition believes that she is better off by deviating from the grand coalition (i.e., no other } \\
\text { coalition blocks the grand coalition and its payoffs). }\end{array}$ \\
\hline Strong Bayesian core & $\begin{array}{l}\text { There exist the payoffs and the grand coalition such that based on each player's beliefs, no player in the } \\
\text { coalition believes that she is better off by deviating from the grand coalition. Moreover, each player who uses } \\
\text { her own view of expected payoffs of other players believes that no other player is better off if she deviates } \\
\text { from the grand coalition. }\end{array}$ \\
\hline
\end{tabular}

types are not known to each player. Moreover, the core is a special case of the weak Bayesian core when there is no type uncertainty (i.e., all the players perfectly know each other's actual type). Since a Bayesian coalitional game generalizes a coalitional game, the Bayesian core may be empty [15].

For an NTU game, the Bondareva-Shapley theorem [21], [23] states that the core of a game is not empty if and only if the game is balanced.

Definition 9. Consider an NTU game. For every $\mathcal{S} \subseteq \mathbb{N}$, let $V_{\mathcal{S}}=V(\mathcal{S}) \times \mathbb{R}^{\mathrm{IN} \backslash \mathcal{S}}$. The NTU game is balanced if

$$
\bigcap_{\forall \mathcal{S} \subseteq \mathbb{N}} V(\mathcal{S}) \subseteq V(\mathbb{N}) .
$$

Observation 1. The weak Bayesian core of the coalitional game for cooperative packet delivery is not empty if

$$
\alpha_{i} R_{i}\left(\mathcal{S}, \overrightarrow{\mathbf{t}}_{\mathcal{S}}^{i}\right)>\beta_{i} C_{i}\left(\mathcal{S}, \overrightarrow{\mathbf{t}}_{\mathcal{S}}^{i}\right), \quad \text { and }
$$

$$
\alpha_{i} R_{i}\left(\mathbb{N}, \overrightarrow{\mathbf{t}}_{\mathrm{IN}}^{i}\right)-\beta_{i} C_{i}\left(\mathbb{N}, \overrightarrow{\mathbf{t}}_{\mathrm{IN}}^{i}\right)>\alpha_{i} R_{i}\left(\mathcal{S}, \overrightarrow{\mathbf{t}}_{\mathcal{S}}^{i}\right)-\beta_{i} C_{i}\left(\mathcal{S}, \overrightarrow{\mathbf{t}}_{\mathcal{S}}^{i}\right)
$$

Proof. Since $\alpha_{i} \geq 0$ and $\beta_{i} \geq 0$, we can find $\alpha_{i}$ and $\beta_{i}$ such that constraint in (15) holds. If the constraint in (15) does not hold, i.e., $u_{i}\left(\mathcal{S}, \overrightarrow{\mathbf{t}}_{\mathcal{S}}^{i}\right)<u_{i}\left(\{i\}, t_{i}\right)=0$, then each mobile node will act alone, and the core is empty. Next, we can express $V(\mathcal{S})$ and $V(\mathbb{N})$ as follows:

$$
\begin{aligned}
V(\mathbb{N})= & \left\{\overrightarrow{\mathbf{x}}^{\mathbb{N}}=\left[\ldots, x_{i}, \ldots\right] \in \mathbb{R}^{\mathbb{I N}} \mid x_{i}\right. \\
\leq & \left.\alpha_{i} R_{i}\left(\mathbb{N}, \overrightarrow{\mathbf{t}}_{\mathbb{N}}^{i}\right)-\beta_{i} C_{i}\left(\mathbb{N}, \overrightarrow{\mathbf{t}}_{\mathbb{N}}^{i}\right), \forall i \in \mathbb{N}\right\} \quad \text { and } \\
V(\mathcal{S})= & \left\{\overrightarrow{\mathbf{x}}^{\mathcal{S}}=\left[\ldots, x_{i^{\prime}}, \ldots\right] \in \mathbb{R}^{\mathcal{S}} \mid x_{i^{\prime}} \leq \alpha_{i^{\prime}} R_{i^{\prime}}\left(\mathcal{S}, \overrightarrow{\mathbf{t}}_{\mathcal{S}}^{i^{\prime}}\right)\right. \\
& \left.-\beta_{i^{\prime}} C_{i^{\prime}}\left(\mathcal{S}, \overrightarrow{\mathbf{t}}_{\mathcal{S}}^{i^{\prime}}\right), \forall i^{\prime} \in \mathcal{S}\right\} .
\end{aligned}
$$

If the constraint in (16) holds, then $\overrightarrow{\mathbf{x}}^{\mathbb{I N}}>\overrightarrow{\mathbf{x}}^{\mathcal{S}} \times \mathbb{R}^{\mathbb{I N} \backslash \mathcal{S}}$, $\forall \mathcal{S} \subseteq \mathbb{N}$, which satisfies the definition of a balanced game. Consequently, the Bayesian core is not empty if the constraints in (15) and (16) above are satisfied.

For example, if $\alpha_{i}=0, \forall i \in \mathbb{N}$, then each mobile node in any coalition has no cost for packet delivery. Intuitively, the expected payoffs of all the mobile nodes will be the highest (i.e., the lowest delivery delay for all the mobile nodes). Then, we can obtain $\mathbb{N} \succ_{i} \mathcal{S}, \forall i \in \mathbb{N}$, and $\forall \mathcal{S} \subseteq \mathbb{N}$. Moreover, the obtained coalitional structure, which contains only the grand coalition, i.e., $\Upsilon=\{\mathbb{N}\}$, is also Nash-stable and individually stable since there is no mobile node $i$ that has an incentive to leave the grand coalition and act alone (i.e., $\mathbb{N} \succ_{i}\{i\}$ ).

Remark 2. From Observation 1, there can be multiple Nashstable coalitional structures which are the solutions of the game and one of them can be the grand coalition. The grand coalition for which the set of associated expected payoffs may or may not be the Bayesian core, may not be reached by the proposed algorithm since the algorithm will terminate when any Nash-stable coalitional structure is achieved. Hence, if the Nash-stable coalitional structure composed of only the grand coalition needs to be obtained, the initial coalitional structure in Algorithm 1 has to be set to the grand coalition. Then, if the grand coalitional structure is not Nash-stable, any other coalitional structure, which is Nash-stable, will be obtained.

The different solution concepts described above for the proposed cooperative packet delivery game are summarized in Table 1.

\section{Dynamic Bayesian Coalitional Game}

In this section, we extend the static Bayesian coalitional game to a multistage dynamic Bayesian coalitional game and propose a distributed algorithm for this game. In this game, a player can update her beliefs (i.e., probabilities) about the types of other players as the game evolves. The update is made based on each player's observations about others' behaviors. When the coalitional game with belief update mechanism is repeatedly played, it will converge to a solution which is the same as the solution that could be obtained when all the information are known (i.e., players' types are known). 


\subsection{Belief Update Mechanism}

Each player (i.e., mobile node) can update her beliefs about the types of other players according to Bayes' theorem [24]. As in Section 2.2, let us consider the situation that mobile node $i$ requests a packet from node $j$. In this case, mobile node $i$ can observe whether node $j$ sends the packet or not. Two cases can happen in this scenario, which we define as $\chi_{i j}\left(\omega_{c}=\Omega_{c}\right)$ and $\chi_{i j}\left(\omega_{n}\right)$. Here $\chi_{i j}\left(\omega_{c}=\Omega_{c}\right)$ denotes the event that mobile node $i$ observes a cooperative acknowledgement $\omega_{c}$ implying that it receives the packet from mobile node $j$ successfully. $\chi_{i j}\left(\omega_{n}\right)$ denotes the event that mobile node $i$ observes a noncooperative acknowledgement $\omega_{n}$ implying that it has not received any packet from mobile node $j$. Given an observation $\chi_{i j}\left(\omega_{c}=\Omega_{c}\right)$ or $\chi_{i j}\left(\omega_{n}\right)$ at the $\tau_{i j}$ th time of observation, where $\tau_{i j} \geq 0$, mobile node $i$ can update its belief probability about mobile node $j$ 's wellbehaved type according to Bayes' theorem as shown in (19) and (20).

$$
p_{i j}^{\tau_{i j}+1}\left(\chi_{i j}\left(\omega_{c}=\Omega_{c}\right)\right)=\frac{p_{i j}^{\tau_{i j}}\left(1-p_{e}\right)}{p_{i j}^{\tau_{i j}}\left(1-p_{e}\right)+\left(1-p_{i j}^{\tau_{i j}}\right)\left(1-\varsigma_{i j}^{\tau_{i j}+1}\right)\left(1-p_{e}\right)} .
$$

$$
p_{i j}^{\tau_{i j}+1}\left(\chi_{i j}\left(\omega_{n}\right)\right)=\frac{p_{i j}^{\tau_{i j}} p_{e}}{p_{i j}^{\tau_{i j}} p_{e}+\left(1-p_{i j}^{\tau_{i j}}\right)\left(\varsigma_{i j}^{\tau_{i j}+1}+\left(1-\varsigma_{i j}^{\tau_{i j}+1}\right) p_{e}\right)} .
$$

If mobile node $j$ is misbehaving, the probability that the mobile node will refuse to deliver packets is $s_{i}$. At the time instant $\tau_{i j}+1$, the belief probability of mobile node $i$ that mobile node $j$ refuses to deliver a packet, when mobile node $j$ is misbehaving, is denoted by $\varsigma_{i j}^{\tau_{i j}+1}$, and can be found by using (21) and (22). Let $\left|\chi_{i j}\left(\omega_{c}=\Omega_{c}\right)\right|,\left|\chi_{i j}\left(\omega_{n}\right)\right|$, and $\left|\chi_{i j}\right|$ denote, respectively, the number of observations of cooperative acknowledgement $\omega_{c}$, noncooperative acknowledgement $\omega_{n}$, and total observations of acknowledgement. In (21), the probability that mobile node $i$ will observe a cooperative acknowledgement $\omega_{c}$ can be expressed as the ratio between $\left|\chi_{i j}\left(\omega_{c}=\Omega_{c}\right)\right|$ and $\left|\chi_{i j}\right|$. In (22), the probability that mobile node $i$ will observe a noncooperative acknowledgement $\omega_{c}$ can be expressed as the ratio between $\left|\chi_{i j}\left(\omega_{n}\right)\right|$ and $\left|\chi_{i j}\right|$. Note that the expression on the left hand side of the equation is the theoretical probability but the expression on the right hand side is the probability computed from the actual observations.

$$
\begin{gathered}
p_{i j}\left(1-p_{e}\right)+\left(1-p_{i j}\right)\left(1-\varsigma_{i j}\right)\left(1-p_{e}\right)=\frac{\left|\chi_{i j}\left(\omega_{c}=\Omega_{c}\right)\right|}{\left|\chi_{i j}\right|} . \\
p_{i j} p_{e}+\left(1-p_{i j}\right)\left(\varsigma_{i j}+\left(1-\varsigma_{i j}\right) p_{e}\right)=\frac{\left|\chi_{i j}\left(\omega_{n}\right)\right|}{\left|\chi_{i j}\right|} .
\end{gathered}
$$

Then, given mobile node $i^{\prime}$ s belief probability $p_{i j}^{\tau_{i j}}$ about mobile node $j^{\prime}$ s well-behaved type and the number of observations $\left|\chi_{i j}\right|^{\tau_{i j}+1},\left|\chi_{i j}\left(\omega_{n}\right)\right|^{\tau_{i j}+1}$, and $\left|\chi_{i j}\left(\omega_{c}=\Omega_{c}\right)\right|^{\tau_{i j}+1}$ at the $\tau_{i j}+1$ th time of observation, mobile node $i$ can estimate its $\varsigma_{i j}^{\tau_{i j}+1}$ as shown in (23) and (24), where $\left|\chi_{i j}\right|^{\tau_{i j}+1}=\tau_{i j}+1$.

$$
\varsigma_{i j}^{\tau_{i j}+1 \dagger}= \begin{cases}\left(\frac{\left(1-p_{e}\right)-\frac{\left|\chi_{i j}\left(\omega_{c}=\Omega_{c}\right)\right|^{\tau_{i j}+1}}{\left|\chi_{i j}\right|^{\tau_{i j}+1}}}{\left(1-p_{i j}^{\tau_{i j}}\right)\left(1-p_{e}\right)}\right), & \left|\chi_{i j}\left(\omega_{c}=\Omega_{c}\right)\right|^{\tau_{i j}+1}>0 \\ \left(\frac{\mid \frac{\mid \chi_{i j}\left(\omega_{n}\right)}{\left|\chi_{i j}\right|^{\tau_{i j}+1}}-p_{e}}{\left(1-p_{i j} \tau_{i j}\right)\left(1-p_{e}\right)}\right), & \left|\chi_{i j}\left(\omega_{n}\right)\right|^{\tau_{i j}+1}>0 .\end{cases}
$$

For mobile node $i$, we obtain the new belief probability that mobile node $j$ will refuse to deliver packet at the $\tau_{i j}+1$ th time of observation, i.e., $\varsigma_{i j}^{\tau_{i j}+1 \dagger}$, in (23). Note that in (23), when both the number of observations of cooperative acknowledgement (i.e., $\left|\chi_{i j}\left(\omega_{c}=\Omega_{c}\right)\right|^{\tau_{i j}+1}$ ) and the number of observations of noncooperative acknowledgement (i.e., $\left|\chi_{i j}\left(\omega_{n}\right)\right|^{\tau_{i j}+1}$ ) are greater than zero, using either the first or the second equation will give the same result. Since the probability obtained at each time of observation is independently calculated using the statistical data (i.e., $\left|\chi_{i j}\right|^{\tau_{i j}+1},\left|\chi_{i j}\left(\omega_{n}\right)\right|^{\tau_{i j}+1}$, and $\left.\left|\chi_{i j}\left(\omega_{c}=\Omega_{c}\right)\right|^{\tau_{i j}+1}\right)$, each mobile node can update its belief probability $\varsigma_{i j}^{\tau_{i j}+1}$ as the weighted sum of the previous value $\varsigma_{i j}^{\tau_{i j}}$ and the new value $\varsigma_{i j}^{\tau_{i j}+1 \dagger}$ as shown in (24) below:

$$
\varsigma_{i j}^{\tau_{i j}+1}=w_{1} \varsigma_{i j}^{\tau_{i j}+1 \dagger}+w_{2} \varsigma_{i j}^{\tau_{i j}}
$$

where $w_{1}$ and $w_{2}$ are adjustable weight constants such that $0<w_{1}<1,0<w_{1} \leq 1$, and $w_{1}+w_{2}=1$. Note that the linear combination in (24) is based on the concept of exponential moving average (EMA) which is a standard method of estimating an unknown parameter [25]. It is used to estimate the belief $\varsigma_{i j}^{\tau_{i j}+1}$ at step $\tau_{i j}+1$ when the instantaneous belief $\varsigma_{i j}^{\tau_{i j}+1 \dagger}$ in (23) is given.

We can compare the solution of the dynamic Bayesian coalitional game with a dynamic Bayesian noncooperative game. In a dynamic Bayesian noncooperative game [18], a combination of strategies and beliefs is a perfect Bayesian equilibrium if

- the beliefs of each information set are updated by Bayes' theorem whenever applicable, and

- the strategy of each player at each information set is optimal or it maximizes her expected payoff with respect to her beliefs given the strategies of all the other players (i.e., this is called sequential rationality).

Then, when the coalitional game is repeatedly played (i.e., in a multistage game), the players' beliefs are updated according to Bayes' theorem and the players make their decisions to leave and join any coalition optimally based on their preferences (i.e., optimal actions with respect to their beliefs given the strategies of all the others) until a (Bayesian) Nash-stable coalitional structure is achieved. Hence, the solution of each subgame, which is Nash-stable coalitional structure, can be compared to the perfect Bayesian equilibrium for a dynamic Bayesian noncooperative game.

\subsection{Distributed Algorithm}

We present an algorithm for dynamically playing a coalitional game with belief update mechanism based on (19)-(24) in Algorithm 2. First, mobile node $i$ initializes the counter for time of observation, and its belief. Then, the coalitional game is played and a Nash-stable coalitional 
TABLE 2

Default Values of Parameters

\begin{tabular}{|c|l|}
\hline Parameter & Description/value \\
\hline Communication range of base station & Radius of $100 \mathrm{~m}$ \\
Communication range of vehicle & Radius of $50 \mathrm{~m}$ \\
Maximum speed on roads & $50 \mathrm{~km} / \mathrm{h}(31.25 \mathrm{mph})$ \\
Vehicle's acceleration & $0.8 \mathrm{~m} / \mathrm{s}^{2}$ \\
Vehicle's deceleration & $4.5 \mathrm{~m} / \mathrm{s}^{2}$ \\
\hline
\end{tabular}

structure is obtained according to Algorithm 1. Each mobile node then updates its beliefs about other mobile nodes' types while it is helping others in packet delivery. When a period of time to do cooperative packet delivery ends, the mobile nodes repeatedly play the coalitional game given their updated beliefs. In this case, if any player is misbehaving, the belief probabilities for other players about this player's well-behaved type will decrease or that of misbehaved type will increase due to the belief update mechanism. When the mobile nodes repeatedly play the coalitional game given their updated beliefs, the algorithm converges to the Nash-stable coalitional structure which is the same as the solution that could be obtained when all the players' types are known.

Algorithm 2. Distributed algorithm for dynamic Bayesian coalitional game with belief update mechanism

1: Mobile node $i$ initializes the counter $\tau_{i j}, \forall j \in \mathbb{N}$ and $j \neq i . \tau_{i j}$ is the $\tau_{i j}$ th time of observation of helping behavior of mobile node $j$ observed by mobile node $i$ (i.e., $\tau_{i j}=0, \forall j$ ).

2: Mobile node $i$ initializes its beliefs $P_{j}^{i}\left(t_{j}=T_{m}\right)=p_{i j}^{\tau_{i j}}$ and $P_{j}^{i}\left(t_{j}=T_{w}\right)=1-p_{i j}^{\tau_{i j}} \forall j \in \mathbb{N}$ and $j \neq i$, where $0<p_{i j}^{\tau_{i j}}<1$

3: The coalition formation algorithm (Algorithm 1) is run until a Nash-stable coalitional structure based on mobile node's preferences with respect to their beliefs is obtained.

4: loop

5: Mobile node $i$ in $\mathcal{S}_{l}^{i} \in \Upsilon$ helps others to deliver packet according to the current stable coalitional structure $\Upsilon$.

6: Mobile node $i$ observes the helping behavior $\chi_{i j}^{\tau_{i j}}$ of mobile node $j$.

7: Mobile node $i$ updates its belief probability of packet delivery to be refused by other mobile node $j$, if mobile node $j$ is a misbehaving node, i.e., $\varsigma_{i j}^{\tau_{i j}+1}\left(\chi_{i j}^{\tau_{i j}}\left(\omega_{n}\right)\right)$, according to (23) and (24).

8: Mobile node $i$ updates its probabilistic belief about another mobile node $j^{\prime}$ s type $p_{i j}^{\tau_{i j}+1}\left(\chi_{i j}^{\tau_{i j}}\right)$ according to (19) and (20).

9: end loop until packet delivery is done or network state changes

10: Go to Step 3.

\section{Performance Evaluation}

We apply the proposed cooperative packet delivery framework in a vehicle-to-roadside (V2R) communications scenario (i.e., the mobile nodes shown in Fig. 1 are vehicles).
TABLE 3

Rates $\left(r_{i j}\right)$ per Second that Each Vehicle Meets Other Vehicles and an RSB on a Road

\begin{tabular}{|c|c|c|c|c|c|}
\hline Rate & RBS & Vehicle 1 & Vehicle 2 & Vehicle 3 & Vehicle 4 \\
\hline RSB & - & 0.0339 & 0.0345 & 0.0299 & 0.0308 \\
Vehicle 1 & 0.0339 & - & 0.0103 & 0.0108 & 0.0104 \\
Vehicle 2 & 0.0345 & 0.0103 & - & 0.0122 & 0.0145 \\
Vehicle 3 & 0.0299 & 0.0108 & 0.0122 & - & 0.0247 \\
Vehicle 4 & 0.0308 & 0.0104 & 0.0145 & 0.0247 & - \\
\hline
\end{tabular}

In such a scenario, data is transferred through the roadside base stations or wireless access points. Each vehicle is equipped with a Wi-Fi transceiver for downloading data when the vehicle is connected to the RBS.

\subsection{Simulation Parameters and Assumptions}

To obtain the parameters on rate of encounters among the vehicles, we use a microscopic road traffic simulation package named "SUMO," an acronym for "Simulation of Urban MObility" [26] and then use MATLAB to analyze the results obtained from the SUMO simulator. Using the parameters in Table 2, the rates obtained for each vehicle to meet other vehicles and an RBS (i.e., $r_{i j}$ ) are shown in Table 3 . The area of the road map is of size $2 \times 2 \mathrm{~km}$ with 121 intersections. An RBS is located at an intersection for every $200 \mathrm{~m}$ in both horizontal and vertical directions, and they are connected by a wired infrastructure. There are 100 vehicles in the area among which four vehicles, namely, vehicles 1 to 4 , are selected to show the performance evaluation results. Each vehicle moves along the shortest path from a random originating position to a random destination position. Based on the simulation results, we verify the assumption that the time interval of a pair of vehicles to encounter each other is exponentially distributed [11]. Fig. 3 shows the cumulative distribution function (CDF) of the time interval between two consecutive encounters of a pair of vehicles observed in the simulation. It is observed that the CDF obtained from simulations well matches that of an exponential distribution with mean of 1 / 0.0145 seconds.

We assume that the type of each of vehicle 1 , vehicle 2, and vehicle 3 is misbehaving with $\varsigma_{1}=0.90, \varsigma_{2}=0.70$, and $\varsigma_{3}=0.50$, respectively. Vehicle $4 \mathrm{~s}$ type is well behaved (i.e., $\left.\varsigma_{4}=0\right)$. Vehicle $i$ initially believes that the types of the other vehicles $j$ are well behaved with probability $p_{i j}=0.99$ and misbehaving with probability $1-p_{i j}=0.01$. The false positive observation error occurs with $p_{e}=0.1$. Also, each

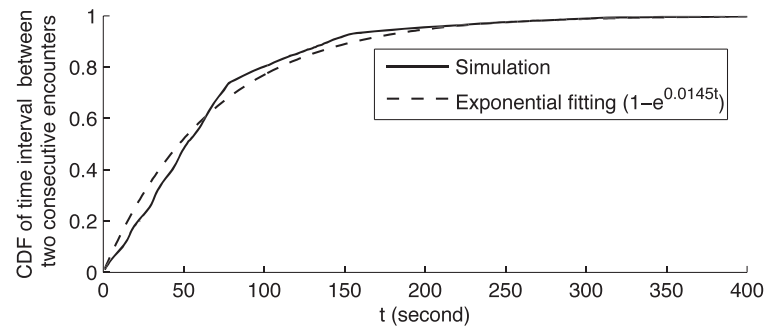

Fig. 3. Cumulative distribution function of the time interval between two consecutive encounters of a pair of vehicles. 
TABLE 4

Fifteen Different Coalitional Structures for Four Vehicles

\begin{tabular}{|c|c|c|c|c|c|}
\hline \multicolumn{5}{|c|}{ Coalitional structure } \\
\hline$\Upsilon_{1}$ & $\{1\},\{2\},\{3\},\{4\}$ & $\Upsilon_{2}$ & $\{1,2\},\{3\},\{4\}$ & $\Upsilon_{3}$ & $\{1\},\{2\},\{3,4\}$ \\
$\Upsilon_{4}$ & $\{1,3\},\{2\},\{4\}$ & $\Upsilon_{5}$ & $\{1\},\{3\},\{2,4\}$ & $\Upsilon_{6}$ & $\{1,4\},\{2\},\{3\}$ \\
$\Upsilon_{7}$ & $\{1\},\{4\},\{2,3\}$ & $\Upsilon_{8}$ & $\{1,2\},\{3,4\}$ & $\Upsilon_{9}$ & $\{1,3\},\{2,4\}$ \\
$\Upsilon_{10}$ & $\{1,4\},\{2,3\}$ & $\Upsilon_{11}$ & $\{1,2,3\},\{4\}$ & $\Upsilon_{12}$ & $\{1,2,4\},\{3\}$ \\
$\Upsilon_{13}$ & $\{1,3,4\},\{2\}$ & $\Upsilon_{14}$ & $\{1\},\{2,3,4\}$ & $\Upsilon_{15}$ & $\{1,2,3,4\}$ \\
\hline
\end{tabular}

vehicle $i$ initially believes that if another vehicle $j$ is misbehaving, it will refuse to deliver a packet with the same probability $\varsigma_{i j}=0.1$. The weight constants of the utility function are assumed to be $\alpha_{1}=\alpha_{2}=\alpha_{3}=\alpha_{4}=15$. A large value of $\alpha$ means that the utility of a vehicle will change significantly when the packet delivery delay changes by a small amount. The weight constants of the cost function are assumed to be $\beta_{1}=\beta_{2}=\beta_{3}=\beta_{4}=1.5$. A small value of $\beta$ (e.g., zero) means that the vehicle does not care about the cost it incurs. We assume that $w_{1}$ and $w_{2}$ (i.e., the weight constants for updating $\varsigma_{i}$ ) are 0.9 and 0.1, respectively. Since there are four vehicles, there are 15 possible coalitions (Table 4). The Time-To-Live value for all packets is assumed to be $d_{i}^{T T L}=35 \mathrm{~s}$. We set the cost of receiving a packet from and forwarding a packet to other vehicles to be equal (i.e., $c_{i j}^{\mathrm{f}}=c_{i j}^{\mathrm{r}}=c_{i}$ ), where $c_{i}$ is referred to as the cost coefficient. This cost coefficient is $c_{i}=1.0$ and assumed to be the same for all the vehicles.

We compare the stable solution from the proposed rational coalition formation game with incomplete information with the solution from optimal coalition formation, and the solution from rational coalition formation with complete information. In the optimal coalition formation, vehicles will form coalitions to maximize the total payoff of all the vehicles given that all the information are completely known. In the case of rational coalition formation with complete information, the coalitions are formed by the vehicles to maximize their individual payoff given that all the vehicles' types are completely known.

\subsection{Numerical Results}

\subsubsection{Nash-Stable Coalitional Structure}

Fig. 4 shows the stationary probabilities of the Nash-stable rational coalition solutions with incomplete information, with complete information, and optimal coalition solution. For the Nash-stable rational coalition formation with

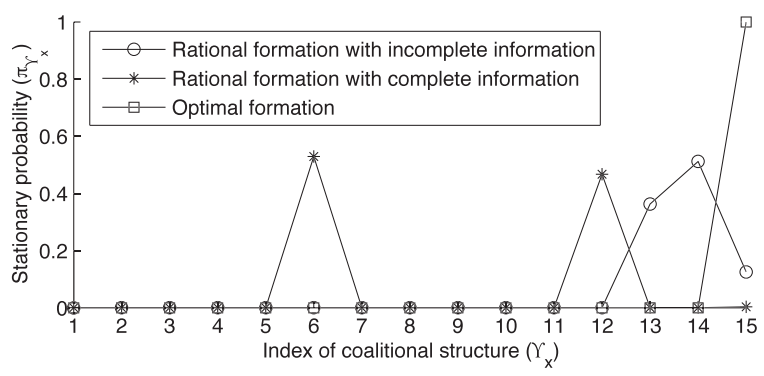

Fig. 4. Stationary probability of the Nash-stable rational coalitional structures with incomplete and complete information and optimal coalitional structure.

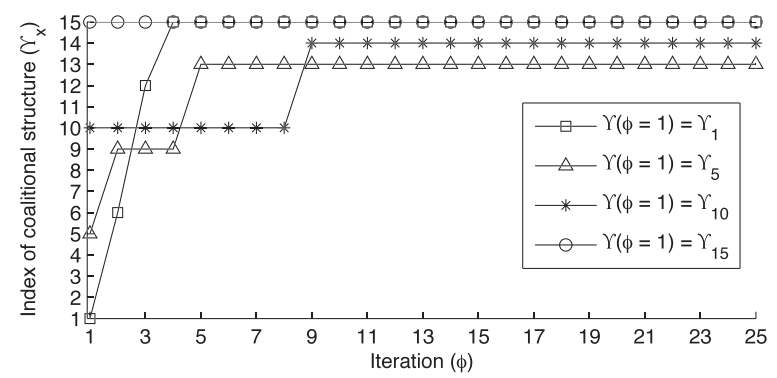

Fig. 5. Nash-stable coalitional structure obtained from the individual preference-based algorithm.

incomplete information, there are three Nash-stable coalitional structures, i.e., $\Upsilon_{13}^{*}=\{\{1,3,4\},\{2\}\}, \Upsilon_{14}^{*}=\{\{1\},\{2,3$, $4\}\}$, and $\Upsilon_{15}^{*}=\{\{1,2,3,4\}\}$. For the Nash-stable rational coalition formation with complete information, there are two Nash-stable coalitional structures, i.e., $\Upsilon_{6}^{*}=\{\{1,4\},\{2,3\}\}$ and $\Upsilon_{12}^{*}=\{\{1,2,4\},\{3\}\}$. For the optimal solution, since the highest total payoff of the optimal coalitional structure is $\Upsilon_{15}^{*}$ the probability of this coalitional structure is one.

Fig. 5 shows the Nash-stable coalitional structure with incomplete information obtained from the individual preference-based algorithm (i.e., Algorithm 1). The initial coalitional structure for each run of the algorithm is set to $\Upsilon_{1}, \Upsilon_{5}, \Upsilon_{10}$, and $\Upsilon_{15}$. When the algorithm runs, we can observe that the coalitional structure changes and then it converges to the Nash-stable coalitional structure, i.e., coalitional structure $\Upsilon_{13}^{*}, \Upsilon_{14}^{*}$, or $\Upsilon_{15}^{*}$ as shown in Fig. 4.

Assuming that the cost coefficient $c_{i}$ is the same for all the vehicles, we vary the cost coefficient $c_{i}$ of all vehicles from 0 to 3 . Figs. $6 \mathrm{a}, 6 \mathrm{~b}$, and $6 \mathrm{c}$ show, respectively, the Nash-stable

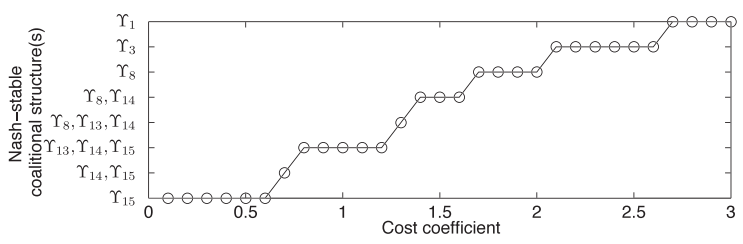

(a)

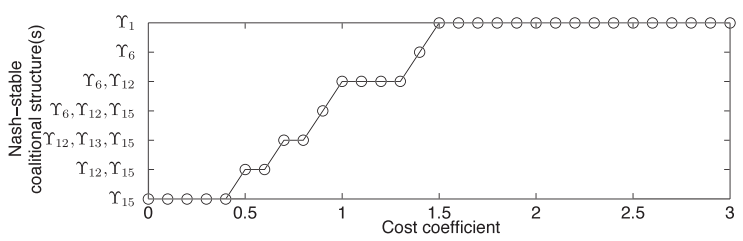

(b)

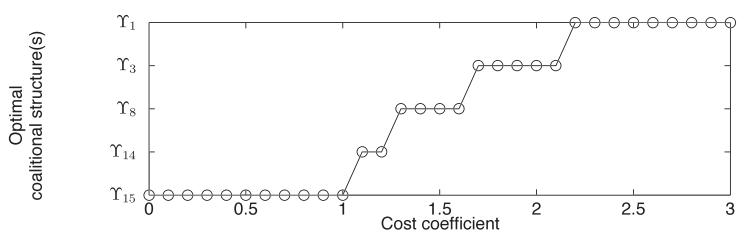

(c)

Fig. 6. (a) Nash-stable coalitional structures with incomplete information, (b) Nash-stable coalitional structures with complete information, and (c) optimal coalitional structures under different values of cost coefficient. 


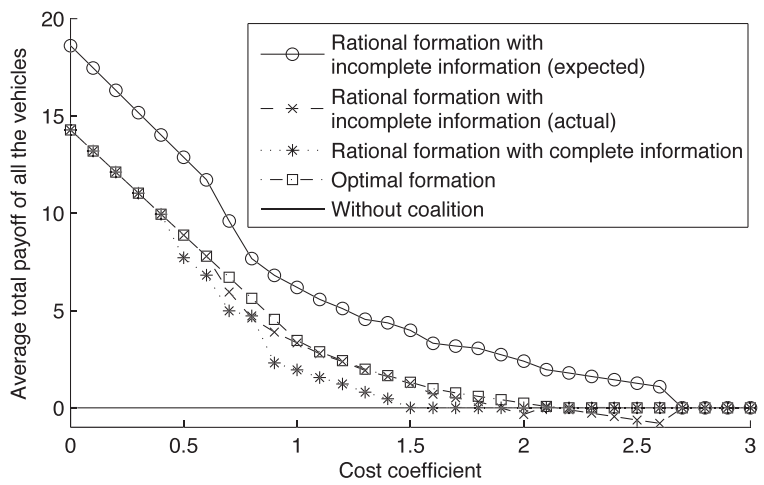

Fig. 7. Total payoff of all the vehicles under different values of cost coefficient.

coalitional structures with incomplete information, with complete information, and the optimal coalitional structure under different values of the cost coefficient. As shown in Fig. 6 , all the vehicles will act alone (i.e., $\Upsilon_{1}^{*}$ is formed) and the total payoff becomes zero when the cost coefficient is greater than 2, 1.4, or 2.1 in the cases of incomplete information, complete information or optimal solution, respectively. Moreover, as shown in Fig. 6a, with incomplete information, the grand coalition and the Bayesian core (i.e., the payoffs of the grand coalition) exist when the cost coefficient is between 0 and 0.6 since there is only one Nashstable coalitional structure $\Upsilon_{15}^{*}$. When the cost coefficient lies between 0.7 and 1.2, the grand coalition may not be formed since it can be blocked by other Nash-stable coalitional structures (e.g., there are two other Nash-stable coalitional structures $\Upsilon_{13}^{*}$ and $\Upsilon_{14}^{*}$ ). Also, as shown in Fig. 6b, with complete information, the grand coalition is Nash-stable when the cost coefficient lies between 0 and 0.4 . When the cost coefficient is between 0.5 and 1.9, the grand coalition may not be formed since other Nash-stable coalitional structures (e.g., $\Upsilon_{6}^{*}, \Upsilon_{12}^{*}$, and $\Upsilon_{13}^{*}$ ) can also be formed.

\subsubsection{Payoffs of the Nodes}

Given all the possible Nash-stable coalitional structures as shown in Figs. 6 and 7 shows the average total payoff of all the vehicles under different values of the cost coefficient. The average total payoff of all the vehicles is obtained as follows:

$$
E\left[u_{\text {total }}\right]=\sum_{i \in \mathbb{N}} \sum_{x=1}^{D_{N}} \pi_{\Upsilon_{x}} u_{i}\left(\mathcal{S}_{l}^{i}\right), \quad \text { for } \quad \mathcal{S}_{l}^{i} \in \Upsilon_{x},
$$

where $D_{N}$ is the $N$ th Bell number and $u_{i}\left(\mathcal{S}_{l}^{i}\right)$ is the payoff of vehicle $i$ when it is a member of coalition $\mathcal{S}_{l}^{i}$.

As expected, for small value of cost coefficient, the average total payoff is high when the coalition is formed. However, when the value of cost coefficient increases, the average total payoff of all vehicles decreases since a higher cost is incurred to all vehicles involved in the cooperative packet delivery. As a result, a vehicle will leave its current coalition if the utility is not higher than the cost incurred from cooperative packet delivery. Moreover, the average total payoff from the optimal solution is equal to or higher than the average total payoffs of the Nash-stable solutions of the rational coalition formations with incomplete and complete information and the payoff when all the players act alone. Note that the Nash-stable solutions of the rational coalition formations with incomplete and complete information are different. Hence, the average payoff of the Nash-stable solution of the rational coalition formation with incomplete information may or may not be higher than that with complete information.

Observation 2. Given a coalitional structure obtained from Algorithm 1, without any belief update, the expected payoff of each vehicle computed under incomplete information is not necessarily lower than the payoff when the vehicle acts alone. However, the payoff actually obtained by each vehicle may be lower than the payoff when the vehicle acts alone.

Discussion. A coalitional structure obtained from Algorithm 1 is guaranteed to be Nash-stable. Consequently, each vehicle believes that its expected payoff is greater than or equal to the payoff when it acts alone. However, the coalitional structure may not be a Nashstable when the complete information scenario is considered. Hence, the vehicle's actual payoff (i.e., the payoff computed based on the actual types of the vehicles after the coalitional structure is formed based on the vehicles' beliefs) may be lower than the payoff when the vehicle acts alone as shown in Fig. 8.

Figs. $8 \mathrm{a}, 8 \mathrm{~b}, 8 \mathrm{c}$, and $8 \mathrm{~d}$ show the average payoffs of vehicles 1, 2, 3, and 4, respectively. We can see that the average payoffs of all vehicles obtained from the proposed game model with incomplete information and with complete information are not lower than zero. In particular, the expected payoff of vehicles at the Nash-stable coalition is not lower than that when they act alone. However, with incomplete information, in some cases, the actual payoff can be lower than zero due to lack of true information about other vehicles' types.

Observation 3. Given a Nash-stable coalitional structure, with the belief update mechanism, each vehicle will obtain the expected payoff close to the actual payoff. Moreover, the actual payoff obtained from the dynamic Bayesian coalitional game can be similar to that from the coalitional game with complete information or at least the actual payoff when the vehicle acts alone.

Discussion. When the Bayes' theorem is used to update the beliefs about the other vehicles in the same coalition, the probabilistic beliefs of the vehicles will converge to the actual values. Then, the expected payoff of each vehicle will converge to its actual payoff. The updated expected payoff may change the Nash-stable coalitional structure. If all of the vehicles' beliefs converge to the actual values, the actual payoffs from the dynamic Bayesian coalitional game will converge to the same values of payoff obtained from the coalitional game with complete information. The results are shown in Figs. 9 and 10. However, if a vehicle has no chance to update its beliefs, its actual payoff may not converge to the payoff obtained from the coalitional game with complete information. In the worst case, the vehicle acts alone after the vehicle can learn some other vehicles' actual types. Hence, the actual payoff is not lower than the payoff when the vehicle acts alone.

\subsubsection{Dynamic Bayesian Coalitional Game}

Given that the grand coalition is formed when the cost coefficient is 1.0, Figs. 9a and $9 \mathrm{~b}$ show the probabilistic beliefs of vehicle 4 when vehicles 1,2 , and 3 are well 


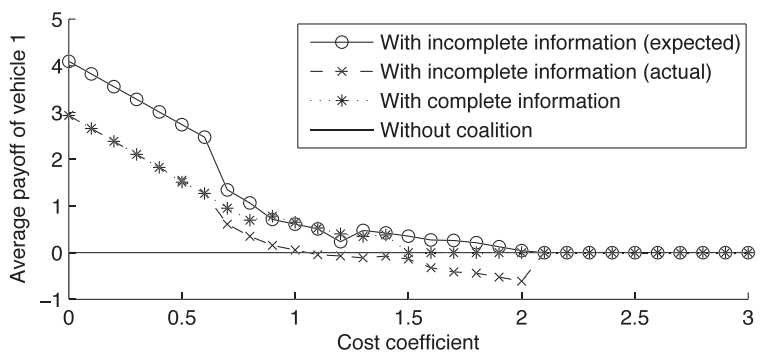

(a)

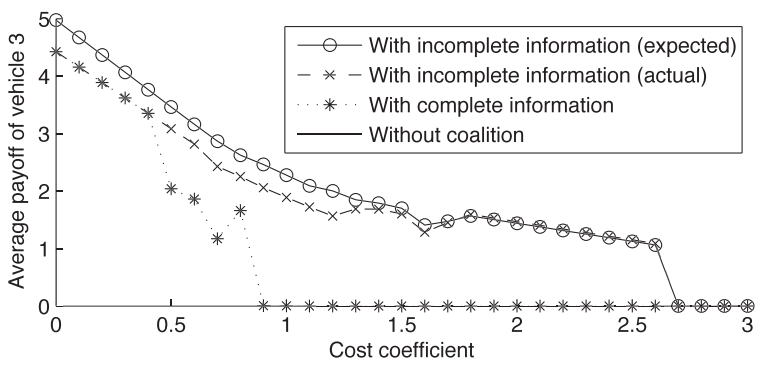

(c)

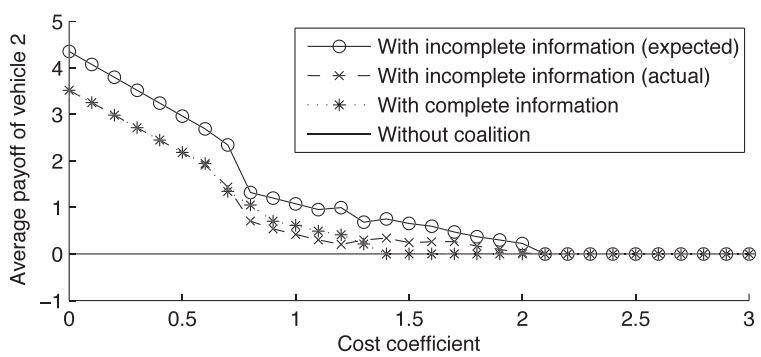

(b)

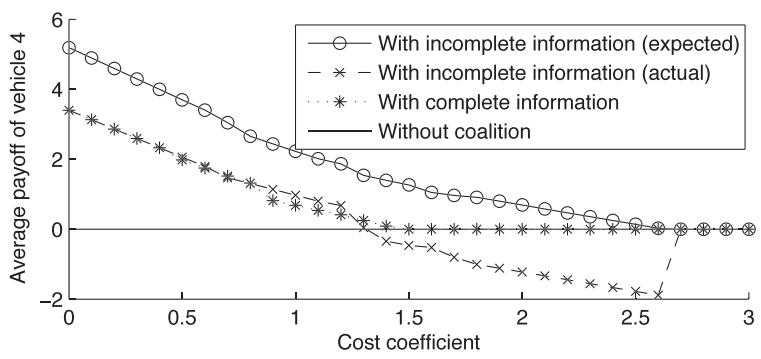

(d)

Fig. 8. Average payoffs obtained from the proposed coalitional game with incomplete information (i.e., both expected and actual payoffs), with complete information, and without coalition: (a) for vehicle 1, (b) for vehicle 2, (c) for vehicle 3, and (d) for vehicle 4.

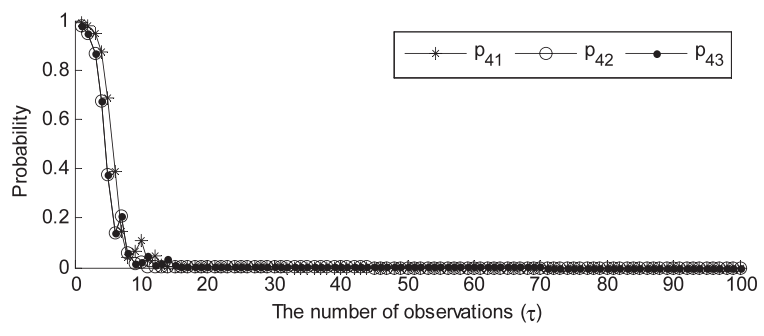

(a)

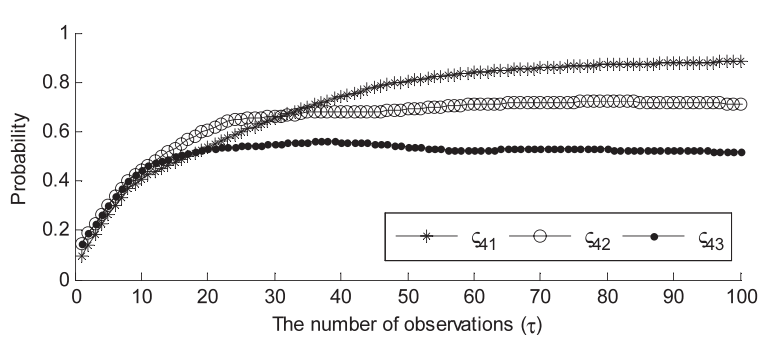

(b)

Fig. 9. (a) Vehicle 4's belief probabilities that vehicles 1,2 , and 3 are well behaved (i.e., $p_{41}, p_{42}, p_{43}$ ), and (b) vehicle 4's belief probabilities that vehicles 1,2 , and 3 will refuse to deliver a packet (i.e., $\left.\varsigma_{41}, \varsigma_{42}, \varsigma_{43}\right)$.

behaved and the probabilistic beliefs of vehicle 4 when vehicles 1,2 , and 3 refuse to deliver a packet, respectively. After vehicle 4 observes the behaviors of vehicles 1, 2, and 3 , it updates its beliefs about the other vehicles' types accordingly. Vehicle 4's belief probabilities that vehicles 1 , 2 , and 3 are well-behaved converge to zero. Also, vehicle 4's belief probabilities that vehicles 1,2 , and 3 will refuse to deliver a packet are close to the actual probabilities (i.e., 0.9, 0.7 , and 0.5 , respectively).

Fig. 10 shows the Nash-stable coalitional structure formed during each period of coalition formation according to Algorithm 2. Given the initial beliefs of all the vehicles and after the first period of coalition formation, with the cost coefficient $0,1,2$, and 3 , the Nash-stable coalitional structures are $\Upsilon_{15}^{*}, \Upsilon_{14}^{*}, \Upsilon_{8}^{*}$, and $\Upsilon_{1}^{*}$, respectively. Each vehicle in the same coalition updates its beliefs during the period of each round of coalition formation. After that, the coalition formation starts its next round. Given that the cost coefficient is 1 , the Nash-stable coalitional structure changes from $\Upsilon_{14}^{*}$ to $\Upsilon_{13}^{*}$, from $\Upsilon_{13}^{*}$ to $\Upsilon_{12}^{*}$, and from $\Upsilon_{12}^{*}$ to $\Upsilon 6^{*}$ at the end of the coalition formation periods $1,2,3$, and 4 , respectively. Given that the cost coefficient is 2, the Nashstable coalitional structure changes from $\Upsilon_{8}^{*}$ to $\Upsilon_{5}^{*}$, from $\Upsilon_{5}^{*}$ to $\Upsilon_{15}^{*}$, and from $\Upsilon_{15}^{*}$ to $\Upsilon_{1}^{*}$, at the end of coalition formation periods 1,2,3, and 4, respectively. When the cost coefficient is 0 and 3 , the Nash-stable coalitional structure does not change. When the cost coefficient is 0 , all the vehicles will always form the grand coalition regardless of the types of all the vehicles. When the cost coefficient is $3, \Upsilon_{1}^{*}$ is first formed, and no vehicle has a chance to update its beliefs. However, with the complete information, the same Nashstable coalitional structure is reached.

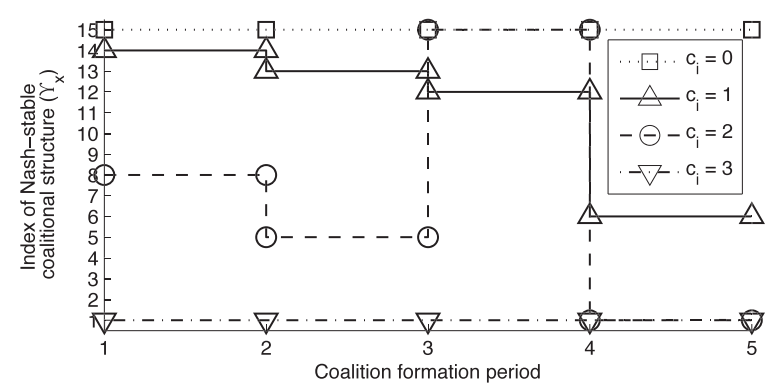

Fig. 10. Nash-stable coalitional structure during each period of coalition formation. 
Comparing the results shown in Figs. 6 and 10, we observe that, the Nash-stable coalitional structure obtained from the dynamic Bayesian coalitional game with incomplete information and the belief update mechanism converges to the same Nash-stable coalitional structure obtained from the coalitional game with complete information. Hence, the actual payoff from the dynamic Bayesian coalitional game is similar to the payoff obtained from the coalitional game with complete information. Moreover, there is a case that a vehicle has no chance to join any coalition with others. For example, with incomplete information of the vehicles' types and given the vehicles' beliefs, the Nash-stable coalitional structure in which all the coalitions are singleton coalitions, i.e., $\Upsilon_{1}^{*}$ is first formed. Then, a vehicle will not have a chance to update its beliefs about the other vehicles' types. With complete information, if the obtained Nash-stable coalitional structure is not the same as that with incomplete information (i.e., it is not $\Upsilon_{1}^{*}$ ), it is not possible that the actual payoff from the Bayesian coalitional game will converge to the payoff obtained from the coalitional game with complete information. However, if a vehicle cannot update all its beliefs, its actual payoff will not be lower than the payoff when it acts alone.

\section{Related Work}

There are only a very few works using the dynamic Bayesian game theory to solve the resource allocation problem in wireless and mobile communications systems. For example, in [27], [28], a multislot coopeartive spectrum sharing mechanism was modeled as a dynamic Bayesian bargaining game. A primary user (PU) offers its licensed spectrum to a secondary user (SU) while the secondary user will relay the primary user's data. However, the information about SU's energy cost is unknown by the PU. A perfect Bayesian equilibrium or sequential equilibrium, which is a refinement of perfect Bayesian equilibrium, can be obtained as the solution of the game.

In our cooperative packet delivery model, we have used a dynamic Bayesian coalitional game. The key idea of the coalitional game theory is to study the formation of coalition among players in a game. In wireless networks, coalitional games have been used to model and analyze the resource allocation problem, where coalitions of mobile nodes are formed so that the mobile nodes achieve higher utilities compared to when they do not form coalitions. In [5], a coalitional game model was used to solve the problem of packet-forwarding among boundary nodes and backbone nodes in wireless networks. As a result, the boundary nodes can transmit their packets effectively. In [6], a coalitional game was proposed for cooperative data service among the base stations in vehicular networks. Through coalitions, the revenue of any cooperative group of base stations can be improved by exploiting the underlying vehicle-to-vehicle content-sharing network. In [7], a coalitional game was proposed for cooperative bandwidth sharing among mobile nodes (i.e., vehicles) in vehicle-to-roadside communications scenarios. When mobile nodes form coalitions, the mobile nodes can reduce the cost of bandwidth reservation while meeting their quality-of-service (QoS) requirements; hence, higher utilities can be achieved.

In reality, it is not guaranteed that the rational mobile nodes, which are the members of the coalition, will agree to always help each other in the same coalition since some of them may misbehave. For packet forwarding in mobile ad hoc networks (MANETs), some reputation-based and game theory-based cooperation enforcement mechanisms were proposed [29], [30], [31] to prevent nodes from misbehaving. However, most of the existing schemes assume that, to detect the misbehaving nodes, perfect observations of mobile nodes' behaviors (e.g., through monitoring systems) are available. There are only a very few work which consider that the nodes' behaviors cannot be perfectly observed. For example, in [31], a noncooperative repeated game with belief-based cooperative enforcement mechanism was presented for packet forwarding in mobile ad hoc networks under imperfect observation.

In order to study the problems of cooperation under uncertainty or imperfect observation of mobile nodes' behaviors or types, coalitional game was generalized to Bayesian coalitional game [15], [16], [17]. In [15] and [17], the Bayesian core, which is a solution concept of the Bayesian coalitional game, was studied. With this concept, no group of players would prefer to leave a grand coalition and form a new coalition. In [15], the notion of Bayesian core in a transferable-utility Bayesian coalitional game was proposed based on how the payoffs can make a grand coalition stable given the beliefs about players' types. In [17], the notion of ex-interim blocking was proposed to achieve the Bayesian core. Ex-interim blocking means that given the beliefs of the players, a grand contract is blocked by a coalition (i.e., a set of expected payoffs in the coalition, which the authors call a contract).

\section{Conclusion}

We have presented a dynamic Bayesian coalitional game for coalition-based cooperative packet delivery among mobile nodes in a mobile network under uncertainty in node behavior (i.e., selfishness of nodes). The mobile nodes are rational to form coalitions to maximize their individual payoffs. Based on the individual preferences, which are related to the expected payoffs of the nodes, a Bayesian coalitional game has been formulated to model the decision making process of mobile nodes (e.g., to cooperatively deliver the packets of other mobile nodes or not). A Nashstable coalitional structure, which is the solution of this coalitional game, can be obtained by using the individual preference-based algorithm. Moreover, a belief update mechanism based on Bayes' theorem has been proposed. With this mechanism, each mobile node can update its beliefs about the other mobile nodes' types (i.e., well behaved or misbehaving) under the proposed Bayesian coalitional game. A comprehensive performance evaluation has been carried out for the proposed Bayesian coalitional game.

\section{REFERENCES}

[1] E.P.C. Jones, L. Li, J.K. Schmidtke, and P.A.S. Ward, "Practical Routing in Delay-Tolerant Networks," IEEE Trans. Mobile Computing, vol. 6, no. 8, pp. 943-959, Nov. 2007.

[2] T. Yamada, R. Shinkuma, and T. Takahashi, "Connectivity and Throughput Enhancement by Inter-Vehicle Packet Relay in Road Vehicle Communication Systems," Proc. IEEE GlobeCom, pp. 1-5, Nov./Dec. 2006.

[3] Y. Yamao and K. Minato, "Vehicle-Roadside-Vehicle Relay Communication Network Employing Multiple Frequencies and Routing Function," Proc. Sixth Int'l Symp. Wireless Comm. Systems, pp. 413-417, Sept. 2009. 
[4] V.N.G.J. Soares, J.J.P.C. Rodrigues, P.S. Ferreira, and A.M.D. Nogueira, "Improvement of Messages Delivery Time on Vehicular Delay-Tolerant Networks," Proc. Int'l Conf. Parallel Processing Workshops, pp. 344-349, Sept. 2009.

[5] Z. Han and H.V. Poor, "Coalition Games with Cooperative Transmission: A Cure for the Curse of Boundary Nodes in Selfish Packet-Forwarding Wireless Networks," IEEE Trans. Comm., vol. 57, no. 1, pp. 203-213, Jan. 2009.

[6] W. Saad, Z. Han, A. Hjørungnes, D. Niyato, and E. Hossain, "Coalition Formation Games for Distributed Cooperation Among Roadside Units in Vehicular Networks," IEEE J. Selected Areas in Comm., Special Issue on Vehicular Comm. and Networks, vol. 29, no. 1, pp. 48-60, Jan. 2011.

[7] D. Niyato, P. Wang, W. Saad, and A. Hjørungnes, "Coalition Formation Games for Bandwidth Sharing in Vehicle-to-Roadside Communications," Proc. IEEE Wireless Comm. and Networking Conf. (WCNC '10), pp. 1-5, Apr. 2010.

[8] Y. Li, Y. Jiang, D. Jin, L. Su, L. Zeng, and D. Wu, "Energy-Efficient Optimal Opportunistic Forwarding for Delay-Tolerant Networks," IEEE Trans. Vehicular Technology, vol. 59, no. 9, pp. 45004512, Nov. 2010.

[9] I.R. Chen, F. Bao, M. Chang, and J.H. Cho, "Trust Management for Encounter-Based Routing in Delay Tolerant Networks," Proc. IEEE GlobeCom, pp. 1-6, Dec. 2010.

[10] S.C. Nelson, M. Bakht, and R. Kravets, "Encounter-Based Routing in DTNs," Proc. IEEE INFOCOM, pp. 846-854, Apr. 2009.

[11] R. Groenevelt, P. Nain, and G. Koole, "The Message Delay in Mobile Ad Hoc Networks," Performance Evaluation, vol. 62, nos. 14, pp. 210-228, Oct. 2005.

[12] T. Spyropoulos, A. Jindal, and K. Psounis, "An Analytical Study of Fundamental Mobility Properties for Encounter-Based Protocols," Int'l J. Autonomous and Adaptive Comm. Systems, vol. 1, no. 1, pp. 440, July 2008

[13] L.M. Feeney and M. Nilsson, "Investigating the Energy Consumption of a Wireless Network Interface in an Ad Hoc Networking Environment," Proc. IEEE INFOCOM, vol. 3, pp. 1548-1557, 2001.

[14] W. Yu and K.J.R. Liu, "Attack-Resistant Cooperation Stimulation in Autonomous Ad Hoc Networks," IEEE J. Selected Area in Comm., vol. 23, no. 12, pp. 2260-2271, Dec. 2005.

[15] G. Chalkiadakis and C. Boutilier, "Bayesian Reinforcement Learning for Coalition Formation under Uncertainty," Proc. Third Int'l Joint Conf. Autonomous Agents and Multiagent Systems, pp. 1090-1097, July 2004.

[16] G. Chalkiadakis, E. Markakis, and C. Boutilier, "Coalition Formation under Uncertainty: Bargaining Equilibria and the Bayesian Core Stability Concept," Proc. Sixth Int'l Joint Conf. Autonomous Agents and Multiagent Systems, pp. 64:1-64:8, May 2007.

[17] S. Ieong and Y. Shoham, "Bayesian Coalitional Games," Proc. 23rd Nat'l Conf. Artificial Intelligence, 2008.

[18] D. Fudenberg and J. Tirole, Game Theory. MIT, 1991.

[19] A. Bogomonlaia and M. Jackson, "The Stability of Hedonic Coalition Structures," Games and Economic Behavior, vol. 38, pp. 201-230, Jan. 2002

[20] W. Saad, Z. Han, M. Debbah, A. Hjørungnes, and T. Basar, "Coalitional Game Theory for Communication Networks: A Tutorial," IEEE Signal Processing Magazine, vol. 26, no. 5, pp. 7797, Sept. 2009.

[21] S.-S. Byun, "A Survey on the Cooperative Game Theoretic Solution Concepts in Wireless Communications," IEEE Comm. Surveys and Tutorials, submitted, 2010.

[22] T. Arnold and U. Schwalbe, "Dynamic Coalition Formation and the Core," J. Economic Behavior and Organization, vol. 49, no. 3, pp. 363-380, Nov. 2002.

[23] B. Peleg and P. Sudhölter, Introduction to the Theory of Cooperative Games. Springer, 2007.

[24] J.S. Milton and J. Arnold, Introduction to Probability and Statistics: Principles and Applications for Engineering and the Computing Sciences. McGraw Hill, 1995.

[25] M. Pourahmadi, Foundations of Time Series Analysis and Prediction Theory. John Wiley \& Sons, 2001.

[26] D. Krajzewicz, G. Hertkorn, C. Rössel, and P. Wagner, "SUMO (Simulation of Urban MObility): An Open-Source Traffic Simulation," Proc. Fourth Middle East Symp. Simulation and Modelling (MESM '02), pp. 183-187, Sept. 2002.
[27] Y. Yan, J. Huang, X. Zhong, and J. Wang, "Dynamic Spectrum Negotiation with Asymmetric Information," Proc. Int'l Conf. Game Theory for Networks (GameNets), Apr. 2011.

[28] Y. Yan, J. Huang, X. Zhong, M. Zhao, and J. Wang, "Sequential Bargaining in Cooperative Spectrum Sharing: Incomplete Information with Reputation Effect," Proc. IEEE GlobeCom, pp. 1-6, 2011.

[29] P. Michiardi and R. Molva, "A Game Theoretical Approach to Evaluate Cooperation Enforcement Mechanisms in Mobile Ad Hoc Networks," Proc. Int'l Symp. Modeling and Optimization in Mobile, Ad Hoc, and Wireless Networks (WiOPT '03), pp. 3-5, 2003.

[30] E. Altman, A.A. Kherani, P. Michiardi, and R. Molva, "Noncooperative Forwarding in Ad-Hoc Networks," Proc. 15th IEEE Int'l Symp. Personal, Indoor and Mobile Radio Comm., 2004.

[31] Z. Ji, W. Yu, and K.J.R. Liu, "A Belief Evaluation Framework in Autonomous MANETs under Noisy and Imperfect Observation: Vulnerability Analysis and Cooperation Enforcement," IEEE Trans. Mobile Computing, vol. 9, no. 9, pp. 1242-1254, Sept. 2011.

[32] J. Rutten, M. Kwiatkowska, G. Norman, and D. Parker, Mathematical Techniques for Analyzing Concurrent and Probabilistic Systems, P. Panangaden and F. van Breugel, eds., vol. 23, Am. Math. Soc., 2004.

[33] K.S. Trivedi, Probability and Statistics with Reliability, Queueing, and Computer Science Applications. Wiley, 2002.

[34] K.S. Trivedi, M. Malhotra, and R.M. Fricks, "Markov Reward Approach to Performability and Reliability Analysis," Proc. Second Int'l Workshop Modeling, Analysis, and Simulation of Computer and Telecomm. Systems, pp. 7-11, Jan./Feb. 1994.

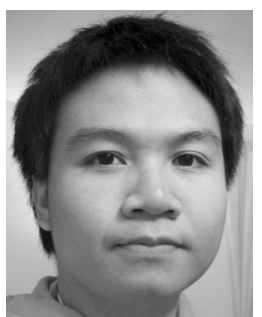

Khajonpong Akkarajitsakul received the BE and $\mathrm{ME}$ degrees in computer engineering from King Mongkut's University of Technology Thonburi, Bangkok, Thailand, in 2005 and 2007, respectively. In 2012, he obtained the $\mathrm{PhD}$ degree in electrical and computer engineering at the University of Manitoba, Winnipeg, MB, Canada His research interests include multiple access in wireless and mobile networks and applied game theory. $\mathrm{He}$ is a member of the IEEE.

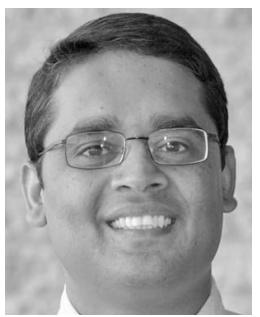

Ekram Hossain received the $\mathrm{PhD}$ degree in electrical engineering from the University of Victoria, Canada, in 2001. He is a full professor in the Department of Electrical and Computer Engineering at the University of Manitoba Winnipeg, Canada. His current research interests include the design, analysis, and optimization of wireless/mobile communications networks and cognitive radio systems (http:// www.ee.umanitoba.ca/ ekram). He currently serves as the editor-in-chief for IEEE Communications Surveys and Tutorials (for the term 2012-2013) and as an editor for the IEEE Journal on Selected Areas in Communications - Cognitive Radio Series and IEEE Wireless Communications. Previously, he served as an area editor for the IEEE Transactions on Wireless Communications in the area of "resource management and multiple access" from 2009-2011 and as an editor for the IEEE Transactions on Mobile Computing from 2007-2012. $\mathrm{He}$ is a registered professional engineer in the province of Manitoba, Canada. He has several research awards to his credit, which include the University of Manitoba Merit Award in 2010 (for research and scholarly activities) and the 2011 IEEE Communications Society Fred Ellersick Prize Paper Award.

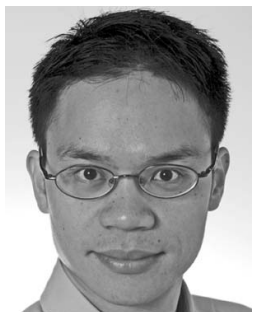

Dusit Niyato received the BE degree from King Mongkut's Institute of Technology Ladkrabang, Bangkok, Thailand, and the PhD degree in electrical and computer engineering from the University of Manitoba, Canada, in 1999 and 2008 , respectively. Currently, he is an assistant professor in the School of Computer Engineering at Nanyang Technological University, Singapore. His research interests include the area of radio resource management in cognitive radio networks, mobile social networks, and mobile cloud computing. 